\title{
Modular First-Order Ontologies via Repositories
}

\author{
Michael GRÜNINGER ${ }^{\mathrm{a}}$, Torsten HAHMANN ${ }^{\mathrm{b}}$, Ali HASHEMI ${ }^{\mathrm{a}}$, Darren ONG $^{\mathrm{a}}$, \\ Atalay OZGOVDE ${ }^{\mathrm{b}}$ \\ ${ }^{a}$ Department of Mechanical and Industrial Engineering, University of Toronto, \\ Toronto, Ontario, Canada M5S $3 G 8$ \\ ${ }^{\mathrm{b}}$ Department of Computer Science, University of Toronto, Toronto, Ontario, Canada \\ M5S $3 G 8$
}

\begin{abstract}
.
From its inception, the focus of ontological engineering has been to support the reusability and shareability of ontologies, as well as interoperability of ontologybased software systems. Among the approaches employed to address these challenges have been ontology repositories and the modularization of ontologies. In this paper we combine these approaches and use the relationships between first-order ontologies within a repository (such as non-conservative extension and relative interpretation) to characterize the criteria for modularity. In particular, we introduce the notion of core hierarchies, which are sets of theories with the same non-logical lexicons and which are all non-conservative extensions of a unique root theory. The technique of relative interpretation leads to the notion of reducibility of a theory to a set of theories in different core hierarchies. We show how these relationships support a semi-automated procedure that decomposes an ontology into irreducible modules. We also propose a semi-automated procedure that can use the relationships between modules to characterize which modules can be shared and reused among different ontologies.
\end{abstract}

Keywords. ontology repository, modularity, first-order logic, non-conservative extension, conservative extension, reducibility, similarity

\section{Introduction}

Ontology repositories have been proposed as part of the infrastructure required to support interoperability of ontology-based software systems through the reusability and shareability of ontologies. We are naturally led to the question as to the modular nature of the constituent ontologies within such a repository. If a repository consists of a set of ontologies, can the repository provide a way of modularizing its constituent ontologies? What criteria do we use to identify which modules are atomic, so that they form the smallest building blocks for assembling new modular ontologies?

For the purpose of this paper, we consider ontologies as axiomatizations of some concept(s) in the language of first-order logic; each ontology gives rise to a theory as the closure of the ontology's axiomatization under logical consequences (entailments). A module of an ontology is then an axiomatization of a subtheory of the ontology. One can take an "internal" view of modules in the sense that an ontology is explicitly con- 
structed by extending and combining existing ontologies in the repository. An alternative "external" view decomposes an ontology based on logical relationships of its subtheories to existing modules in the repository. In this paper we explore what logical relationships among a set of first-order ontologies can be used as basis for the decomposition of ontologies following this external view and how these relationships can be used. We identify two levels of modularity that arise from these logical relationships.

One fundamental logical relationship is non-conservative extension; this leads in Section 2 to the notion of a hierarchy of ontologies that have the same non-logical lexicon. The resulting first approach to modularity decomposes an ontology into atomic modules in a single hierarchy based on similarities and differences between the ontology and existing atomic modules in that hierarchy. Each ontology in a hierarchy is then logically equivalent to a set of atomic modules.

For ontologies that are axiomatized using disjoint non-logical lexicons ${ }^{1}$, we need to determine whether or not the non-logical lexicon of one ontology can be interpreted in the non-logical lexicon of other ontologies in the repository. This leads in Section 3 to our second approach to modularity, based on the relationship of reducibility, in which one ontology is definably equivalent to the union of existing modules in different hierarchies.

We define a repository to be a set of hierarchies that are ordered by the reducibility relationship; the hierarchies whose constituent modules are not all reducible to other modules in the repository are the core hierarchies and they form the modular building blocks of the ontologies in the repository. Each core hierarchy has a common root theory that captures inherent properties of the axiomatized concept. E.g. all ontologies in a repository that axiomatize the notion of "time" are some kind of linear order. Each atomic module non-conservatively extends the root theory but no other theory in the hierarchy, and specifies an ontological design choice, which captures a property of all its models not commonly accepted by all ontologies within the same hierarchy. E.g. there are time ontologies that are dense and others that are discrete (and as such non-dense), so density is an ontological design choice for time ontologies. In this pragmatic approach not all theoretically feasible ontological design choices are extracted, but only the ones that are used by at least one of the ontologies in the hierarchy. We can then view each ontology contained in a single core hierarchy of the repository as an axiomatization of the root theory of the hierarchy extended by a set of additional ontological design choices. Thereby, we give a more formal perspective on the informal notion of design choices.

We consider repositories to be dynamic ontology design artefacts, that is, the modularization of the constituent ontologies evolves with the addition of more ontologies. We propose semi-automated procedures for the decomposition and refinement of ontologies that rely on the existing modules in the repository, giving practical guidance for modularizing ontologies and designing new modular ontologies. The set of procedures in Section 6 identify modules of an ontology that are all equivalent to modules in core hierarchies which provides a modularization of the ontology. The set of procedures in Section 7 update a single hierarchy with a new ontology that has the same non-logical lexicon. The new ontology is decomposed into a set of independent ontological design choices based on the other modules in that hierarchy. Through these procedures previously atomic ontological design choices may be split into a set of more fine-grained design choices and completely new design choices may be introduced. These procedures

\footnotetext{
${ }^{1}$ If ontologies do not use exactly the same non-logical lexicons but share some non-lexical symbols, those shared can be easily renamed to ensure that the ontologies use strictly disjoint non-logical lexicons.
} 
relate closely to the two kinds of modularity. The first set of procedures divide a new ontology into modules in different hierarchies while the second set of procedures divide those modules into atomic modules within each hierarchy.

\section{Hierarchies}

Before we define what it means for a set of ontologies (or their resulting theories) to be in a hierarchy, we introduce some preliminary notions that are used throughout the paper. These are well-known definitions in mathematical logic 2 .

Definition 1 A first-order theory is a set of first-order sentences closed under logical entailment.

Definition 2 Let $T$ be a first-order theory.

The non-logical lexicon (signature) of $T$, denoted by $\Sigma(T)$, is the set of all constant symbols, function symbols, and relation symbols that are used in $T$.

The language of $T$, denoted by $\mathcal{L}(T)$, is the set of all first-order formulas that only use the non-logical symbols in the signature $\Sigma(T)$.

Definition 3 Let $T_{1}, T_{2}$ be two first-order theories such that $\Sigma\left(T_{1}\right) \subseteq \Sigma\left(T_{2}\right)$.

We say that $T_{2}$ is an extension of $T_{1}$ iff for any sentence $\sigma \in \mathcal{L}\left(T_{1}\right)$,

$$
\text { if } \quad T_{1}=\sigma \quad \text { then } \quad T_{2}=\sigma .
$$

$T_{2}$ is a conservative extension of $T_{1}$ iff for any sentence $\sigma \in \mathcal{L}\left(T_{1}\right)$,

$$
T_{2} \models \sigma \quad \text { iff } \quad T_{1}=\sigma .
$$

$T_{2}$ is a non-conservative extension of $T_{1}$ iff $T_{2}$ is an extension of $T_{1}$ and there exists a sentence $\sigma \in \Sigma\left(T_{1}\right)$ so that

$$
T_{1} \not \models \sigma \quad \text { and } \quad T_{2} \models \sigma .
$$

A first-order ontology is a set of first-order sentences (axioms) that characterize a first-order theory, which is the closure of the ontology's axioms under logical entailment. In the rest of the paper we will simply drop the term first-order and assume ontologies and theories to be first-order.

Two ontologies $O_{1}$ and $O_{2}$ that use the same non-logical lexicon $\Sigma$ have logically equivalent theories if for all sentences $\sigma$ expressed in $\Sigma$

$$
O_{1} \models \sigma \Longleftrightarrow O_{2}=\sigma
$$

We do not distinguish ontologies with logically equivalent theories. Even though ontologies stored in an actual repository are concrete sets of axioms, we will discuss our ideas in this paper in terms of the more abstract repository of theories. In other words,

\footnotetext{
${ }^{2}$ For additional background for the notions of structures and models, we refer the reader to [17].
} 
the concepts described in this paper do not depend on how the theories are axiomatized. Moreover, we do not distinguish logically equivalent theories with the same nonlogical lexicon. Thus, for the purposes of this paper we assume that each ontology can be uniquely mapped to a theory and vice versa and that two logically equivalent theories are identified by a unique ontology.

We also do not attempt to formally define the notion of an ontology. Although every module is a set of axioms, not every module may be seen as an ontology from the point of view of the modeller. Semantically, a module can simply be a reusable building block for an ontology without capturing a meaningful metaphysical notion in its own right.

Now, the first step is to define sets of theories of the same concept which can be characterized by combinations of ontological design choices. Two theories can then be distinguished by their different sets of design choices. For example, the theory $T_{l p \_d e n s e}{ }^{3}$ consists of the two atomic design choices of the order being linear dense. Each such choice may manifest itself in multiple alternative sets of axioms; there may not be a oneto-one mapping between design choices and concrete axioms of an ontology. While each design choice may be written as an axiom - a so-called logical choice, not every logical choice is an ontological design choice in its own right. The objective is to support the comparability of theories within a hierarchy with respect to their ontological choices, and in this way to explicitly identify their similarities and differences (the axioms in which they differ from all the theories they extend). In this section we will formally define the notions of hierarchy, root theory, trunk theory, similarity, and difference.

\subsection{The Role of Non-conservative Extensions}

If theories are characterized by sets of design choices, they can be ordered by the presence or absence of those design choices. In order for the design choices to be comparable, their axiomatizations need to be expressed using the same signature. Using these intuitions, we can define an ordering over a set of theories:

Definition 4 A hierarchy $\mathbb{H}=\langle\mathcal{H}, \leq\rangle$ is a partially ordered, finite set of theories $\mathcal{H}=$ $T_{1}, \ldots, T_{n}$ such that

1. $\Sigma\left(T_{i}\right)=\Sigma\left(T_{j}\right)$, for all $i, j$;

2. $T_{1} \leq T_{2}$ iff $T_{2}$ is an extension of $T_{1}$;

3. $T_{1}<T_{2}$ iff $T_{2}$ is a non-conservative extension of $T_{1}$.

Hierarchies are obviously not closed under subtheories since infinitely many subtheories exist but we can define the following notion:

Definition 5 Let $\mathbb{H}=\langle\mathcal{H}, \leq\rangle$ be a hierarchy. We call a theory $T$ compatible with the hierarchy $\mathbb{H}$ iff $\Sigma(T)=\Sigma\left(T_{i}\right)$ for any $T_{i} \in \mathcal{H}$.

Compatible theories may not be in the hierarchy, but all theories in a hierarchy are also compatible with the hierarchy. We must also be aware that for a given theory $T$ in

\footnotetext{
${ }^{3}$ We adopt the following naming convention for the online CLIF axiomatization of theories each theory is associated with a URI such that the subscript in the theory name is the unqualified name in the URI. For example, the CLIF axiomatization of the theory $T_{l p_{-} \text {dense }}$ can be found at http://stl.mie.utoronto.ca/colore/time/lp-dense.clif
} 


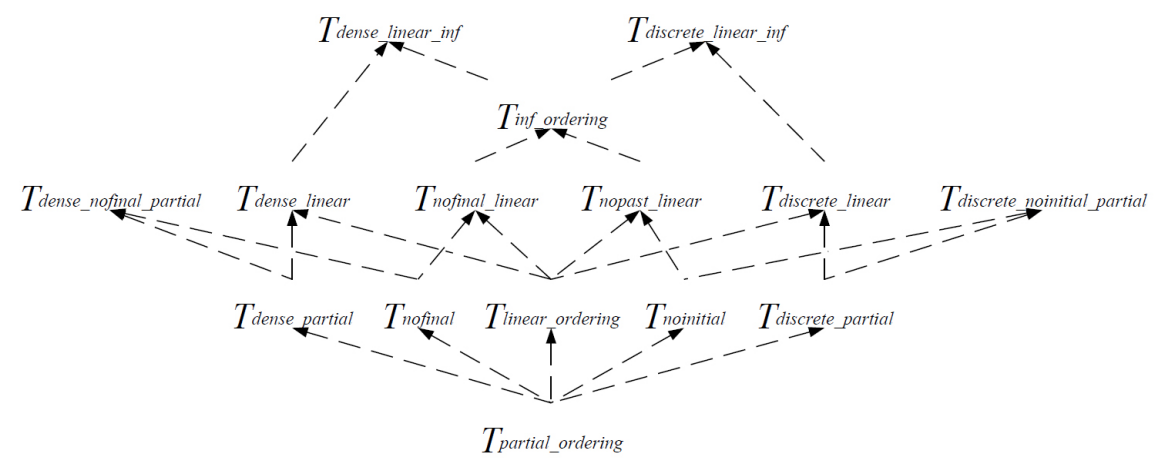

Figure 1. Hierarchy of Ordering theories. Dashed lines denote non-conservative extensions.

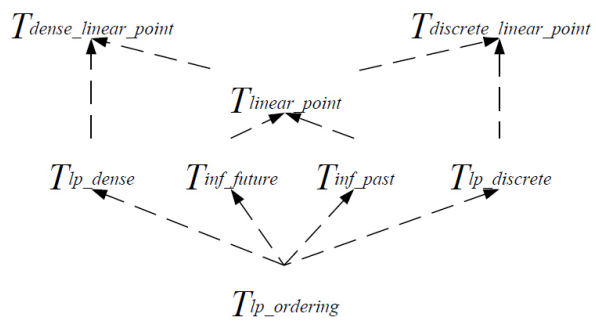

Figure 2. Hierarchy of Timepoint theories. Dashed lines denote non-conservative extensions.

a hierarchy, a subtheory $S$ of $T$ in the hierarchy may not be axiomatizable by a subset of the axioms of $T$. In other words, the partial order over theories within an hierarchy cannot be established solely by relying on the subset relations between sets of axioms.

Consider the hierarchy of ordering theories shown in Figure 1, with theories ordered such that ones higher in the hierarchy non-conservatively extend those they are connected to below. For example, $T_{\text {linear_ordering }}$ is the theory of linearly ordered points and $T_{\text {dense_linear }}$ is the theory of dense linearly ordered points, so following Definition 1 we have $T_{\text {dense_linear }}>T_{\text {linear_ordering }}$ since every sentence entailed by $T_{\text {linear_ordering }}$ is also entailed by $T_{\text {dense_linear }}$ and there exist sentences entailed by $T_{\text {dense_linear }}$ that are not entailed by $T_{\text {linear_ordering. Similarly, for the hierarchy of timepoint theories }}$ shown in Figure 2, we have $T_{\text {dense_linear_point }}>T_{l p_{-} \text {dense }}$ since every sentence entailed by $T_{l p \_d e n s e}$, the theory of dense linearly ordered timepoints, is also entailed by $T_{\text {dense_linear_point }}$, the theory of dense linearly ordered timepoints that extends infinitely into the past and future, but not vice versa.

Although the ordering over theories in a hierarchy is defined with respect to entailment, the two notions are distinct. For example, consider the standard theory $T h(\mathcal{N}, 0, S)$ of natural numbers vs. the theory $T h(\mathcal{N}, 0, S, l t)$ of natural numbers with an additional ordering relation $l t$. We have

$$
\operatorname{Th}(\mathcal{N}, 0, S, l t) \models T h(\mathcal{N}, 0, S)
$$

yet we do not have $T h(\mathcal{N}, 0, S, l t) \leq T h(\mathcal{N}, 0, S)$, since these two theories do not share a signature and hence cannot be in the same hierarchy. 
Let $\operatorname{Mod}(T)$ denote the set of models of a theory $T$. Given two theories $T_{1}$ and $T_{2}$ such that $T_{2} \models T_{1}$, we do not in general have $\operatorname{Mod}\left(T_{2}\right) \subseteq \operatorname{Mod}\left(T_{1}\right)$, since the models of $T_{2}$ may not be models of $T_{1}$. For example, suppose that $T_{1}$ is the theory of bipartite incidence structures and $T_{2}$ is the theory of ordered geometries. Models of $T_{2}$ are not isomorphic to models of $T_{1}$; rather, they contain substructures that are isomorphic to models of $T_{1}$. On the other hand, if we restrict ourselves to theories within the same hierarchy, then we can use subsets of models to characterize the relationships between theories:

Lemma 1 If $T_{1}$ and $T_{2}$ are theories in the same hierarchy, then

$$
T_{1}<T_{2} \Leftrightarrow \operatorname{Mod}\left(T_{2}\right) \subsetneq \operatorname{Mod}\left(T_{1}\right) .
$$

Proof: $\Rightarrow$ : Assume that $T_{1}<T_{2}$. Now suppose there exists a model $\mathcal{M} \in \operatorname{Mod}\left(T_{2}\right)$ that is not a model of $T_{1}$, i.e. $\mathcal{M} \notin \operatorname{Mod}\left(T_{1}\right)$. There exists a sentence $\sigma$ so that $\sigma$ is satisfied by $\mathcal{M}$ and $\sigma$ is not consistent with $T_{1}$, i.e. $T_{1} \models \neg \sigma$. Since $T_{2} \not \models \neg \sigma$, $T_{1} \nless T_{2}$, a contradiction.

Moreover, $T_{2} \not \leq T_{1}$, i.e. there exists a sentence $\sigma$ such that $T_{2} \models \sigma$ but $T_{1} \not \models \sigma$. There exists a model of $T_{1}$ that satisfies $\neg \sigma$ but which cannot be a model of $T_{2}$. Hence $\operatorname{Mod}\left(T_{2}\right) \subsetneq \operatorname{Mod}\left(T_{1}\right)$.

$\Leftarrow$ : If $T_{1} \not \leq T_{2}$, then there exists a sentence $\sigma$ such that $T_{1} \models \sigma$ but $T_{2} \not \models \sigma$. A model of $T_{2}$ exists that satisfies $\neg \sigma$ but cannot be a model of $T_{1}$. This contradicts the assumption that $\operatorname{Mod}\left(T_{2}\right) \subsetneq \operatorname{Mod}\left(T_{1}\right)$.

Moreover, there must be a model of $T_{1}$ that is not a model of $T_{2}$. Let $\sigma$ capture this model which is consistent with $T_{1}$ but not consistent with $T_{2}$. Thus, $T_{2} \models \neg \sigma$ while $T_{1} \not \models \neg \sigma$, hence $T_{2} \not \leq T_{1}$.

In other words, models of a theory in a hierarchy are models of its subtheories that are in the same hierarchy; extensions only restrict the sets of models, not the structures of the models themselves. This is not in general the case for all theories; for example, the theories in the PSL Ontology [12] do not form a hierarchy, since each theory expands the non-logical lexicon; as a result, the models of a theory such as the PSL Ontology are constructed by combining and extending models of other theories.

Root theories are the theories that do not extend any other theory in the hierarchy.

Definition 6 A theory $T$ in a hierarchy is a root theory iff it does not non-conservatively extend any other theory in the same hierarchy.

In the ordering hierarchy, $T_{\text {partial_ordering }}$ is the only root theory as it does not extend any other theory in its hierarchy and for the timepoints hierarchy the only root theory is $T_{l p \_o r d e r i n g}$ for the same reason. Though in general multiple root theories may exist, we later define a more constrained type of hierarchy - a closed hierarchy - that requires the root theory to be uniquely determined up to logical equivalence.

For two theories $T_{1}, T_{2}$ with the same signature $\Sigma$ we say that $T_{2}$ is stronger than $T_{1}$ (or, equally, that $T_{1}$ is weaker than $T_{2}$ ) if $T_{2}$ is a non-conservative extension of $T_{1}$ independent of whether $T_{1}$ and $T_{2}$ are actually contained in a hierarchy. Obviously, if $T_{2}>T_{1}$ for theories $T_{1}, T_{2}$ in the same hierarchy, then $T_{2}$ is stronger than $T_{1}$. 


\subsection{Similarity between Theories in a Hierarchy}

Within a hierarchy, all theories are partially ordered by their sets of theorems. In the following we want to extract a specific subset $S$ of the theorems shared by two theories $T_{1}$ and $T_{2}$ within a hierarchy. We refer to this subset of shared inferred sentences, which itself is a theory with signature $\Sigma\left(T_{1}\right)$, as the similarity of $T_{1}$ and $T_{2}$, while theorems that $T_{1}$ and $T_{2}$ differ in are captured as remainders (with respect to the similarity $S$ ). Again, this notion of similarity is defined with respect to subtheories and not with respect to sets of axioms; however, it still varies from the idea discussed in [15].

Obviously, two theories in the same hierarchy have a common strongest subtheory (up to logical equivalence). However, this subtheory may be overly specific. Consider for example the theories $T_{\text {nofinal }}$ and $T_{\text {noinitial }}$ in the hierarchy of ordering theories in Fig. 1:

$$
\begin{aligned}
T_{\text {nofinal }} & \models \forall x \exists y(x<y) \\
T_{\text {noinitial }} & \models \forall x \exists y(y<x)
\end{aligned}
$$

Either sentence corresponds to some intuitive design choice for orderings: whether there is some initial (smallest) element and whether there is some final (largest) element. This is maybe better illustrated by the same entailments in the two theories of timepoints $T_{\text {inf_future }}$ and $T_{\text {inf_past }}$. In some applications (such as planning) it may be suitable to assume that for every timepoint in the future there exists another timepoint even further in the future. In an application that only reasons about the past (such as dating of ancient artefacts) such assumption may not be useful, but instead we may want to assume that there is a timeline that reaches infinitely into the past. Those two assumptions are independent design choices. Thus their shared theory should make no claim at all about whether a smallest (or earliest) or greatest (latest) element exists. In particular, the similarity of $T_{\text {nofinal }}$ and $T_{\text {noinitial }}$ (and likewise of $T_{\text {inf_future }}$ and $T_{\text {inf_past }}$ ) should not entail the disjunctive sentence

$$
[\forall x \exists y(x<y)] \vee[\forall x \exists y(y<x)]
$$

even though it is entailed by both $T_{\text {nofinal }}$ and $T_{\text {noinitial }}$. The reason is that $T_{\text {nofinal }}$ and $T_{\text {noinitial }}$ already entail disjoint stronger sentences which better capture their respective ontological choices. Hence, our definition of similarity is not simply the strongest subtheory of two given theories in the same hierarchy:

Definition 7 Let $T_{1}$ and $T_{2}$ be theories in the same hierarchy with the signature $\Sigma$.

The similarity between $T_{1}$ and $T_{2}$ is the strongest theory (up to logical equivalence) $S \subseteq T_{1} \overline{\cap T_{2} \text { with }} \Sigma(S)=\Sigma\left(T_{1}\right)$ so that for all $\sigma, \omega \in \mathcal{L}\left(T_{1}\right)$ if

$$
T_{1}=\sigma \quad \text { and } \quad T_{2} \models \omega \quad \text { and } \quad S \not \models \sigma \quad \text { and } \quad S \not \models \omega
$$

then either $\sigma \vee \omega$ is independent of $S$ or $\sigma \vee \omega$ is a tautology.

In other words, $S$ contains all sentences shared by $T_{1}$ and $T_{2}$ except disjunctive sentences $\sigma \vee \omega$ so that $T_{1}$ entails exactly one of $\sigma$ and $\omega$ while $T_{2}$ entails only the 
other. In this sense, we rid the similarity from any unnecessarily specific new disjunctive sentences. In our previous example, choose $T_{1}=T_{\text {nofinal }}, T_{2}=T_{\text {noinitial }}$, $\sigma=\forall x \exists y(x<y)$, and $\omega=\forall x \exists y(y<x)$. Since $T_{1} \models \sigma, T_{2} \not \models \sigma, T_{2} \models \omega$, and $T_{1} \not \models \omega$, the sentence $(*)$ cannot be entailed by the similarity $S$ since it is not a tautology and thus must be independent of $S$. Intuitively, the similarity of $T_{\text {nofinal }}$ and $T_{\text {noinitial }}$ is the common subtheory that also allows models without greatest element and without initial element.

We now confirm that a similarity always exists for two theories in the same hierarchy. Then, by the Definition 7 the similarity must be unique.

Lemma 2 The similarity between two theories $T_{1}, T_{2}$ in the same hierarchy exists.

Proof: Suppose there does not exist a similarity between $T_{1}$ and $T_{2}$. This can be the case only in one of the two following cases. (1) If $T_{1}$ and $T_{2}$ have no common subtheory, or (2) if $T_{1}$ and $T_{2}$ have two subtheories $S$ and $S^{\prime}$ satisfying the last condition of Def. 7 but neither one is stronger than the other.

(1) If no stronger theory satisfies the definition of similarity, then the empty theory ${ }^{4}$, which is unique, is the strongest subtheory of $T_{1}$ and $T_{2}$.

(2) We will derive a contradiction for this case. It requires that two sentences $\sigma, \omega \in \mathcal{L}\left(T_{1}\right)$ exists such that $S \vDash \sigma, S^{\prime} \not \models \sigma, S^{\prime} \vDash \omega, S \not \models \omega$, and

$$
S \equiv\left(S \cap S^{\prime}\right) \cup \sigma
$$

and

$$
S^{\prime} \equiv\left(S \cap S^{\prime}\right) \cup \omega
$$

Because $S \vDash \sigma$ and $S \subseteq T_{1} \cap T_{2}$ the sentence $\sigma$ and any disjunction of the form $\sigma \vee \tau$, where $\tau$ is an arbitrary sentence in the language $\mathcal{L}\left(T_{1}\right)$, do not need to be independent of $S^{\prime} \subseteq T_{1} \cap T_{2}$ and, thus, $\sigma \in S^{\prime}$ by Def. 7. This contradicts our initial assumption that $S^{\prime} \not \models \sigma$. Likewise, we can argue that $\omega \in S$, which contradicts $S \not \models \omega$. Therefore we must have

$$
S \equiv\left(S \cap S^{\prime}\right) \cup \sigma \cup \omega \equiv\left(S \cap S^{\prime}\right) \cup \omega \cup \sigma \equiv S^{\prime} .
$$

Hence, a strongest theory as defined by Def. 7 is unique and thus the similarity always exists for two theories in the same hierarchy.

Note that for general hierarchies, the similarity of two theories in the same hierarchy is not necessarily contained in the hierarchy. Only in closed hierarchies as defined in Section 2.3 , the similarity must be itself a theory of the hierarchy.

Note further that two inconsistent theories in the same hierarchy do not necessarily have the empty theory as similarity. For example, the theory $T_{\text {dense_linear }}$ of dense linearly ordered points and the theory $T_{\text {discrete_linear }}$ of discrete linearly ordered points are inconsistent. Whil $T_{\text {dense_linear }}$ entails density between points, $T_{\text {discrete_linear }}$ entails discreteness between points. Their similarity is the strongest subtheory of both

\footnotetext{
${ }^{4}$ The 'empty theory' contains only first-order validities, but no other sentences.
} 
$T_{\text {dense_linear }}$ and $T_{\text {discrete_linear }}$ that does neither entail density nor discreteness. This theory, $T_{\text {linear_ordering }}$, is one of linearly ordered points independent of the notions of density or discreteness following Def. 7. Note that the similarity cannot always be constructed as the intersection of the axioms of two ontologies.

\subsection{Closed Atomistic Hierarchies}

We can now use the notion of similarity to define when a hierarchy is closed and show that a closed hierarchy has a unique root theory.

Definition 8 A hierarchy $\mathbb{H}=\langle\mathcal{H}, \leq\rangle$ is closed iff the similarity $S$ between any two theories $T_{1}, T_{2} \in \mathcal{H}$ is non-empty and $S \in \mathcal{H}$.

Since a hierarchy is a partially ordered set, this basically states that a closed hierarchy is a meet-semilattice, that is, the meet (similarity) of any two theories in the hierarchy is itself in the hierarchy. The following verifies that a closed hierarchy has a unique smallest element, the so-called root theory.

Lemma 3 A closed hierarchy has a unique root theory up to logical equivalence.

Proof: Suppose two non-logically equivalent roots $T_{1}$ and $T_{2}$ exist in a hierarchy $\mathcal{H}$. Since the hierarchy is closed, the similarity $S$ between $T_{1}$ and $T_{2}$ is non-empty and in $\mathcal{H}$. Both $T_{1}$ and $T_{2}$ extend $S$, thus $S$ is the new root theory while $T_{1}$ and $T_{2}$ cannot be root theories in the hierarchy.

Intuitively, the root theory of a hierarchy axiomatizes an elementary class of structures; each theory in the hierarchy axiomatizes a subclass of this class of structures. For example, the theories that axiomatize classes of (non-strict) partial orderings form a hierarchy - all theories have the same signature (i.e. the ordering relation $\leq$ ) and all are extensions of the theory that contains the three axioms for a non-strict partial ordering (transitivity, reflexivity, and antisymmetry).

Definition 9 Let $\langle\mathcal{H}, \leq\rangle$ be a hierarchy with $T_{1}, T_{2} \in \mathcal{H}$. If $T_{1}<T_{2}$ and no other theory $T \in \mathcal{H}$ exists so that $T_{1}<T<T_{2}$, we say $T_{2}$ covers $T_{1}$ and write $T_{1} \prec T_{2}$.

Since a closed hierarchy contains a unique root theory, the class of theories that cover the root theory will play a key role.

Definition 10 A trunk theory $T$ is a theory that covers the unique root theory of a closed hierarchy $\langle\mathcal{H}, \leq\rangle$.

The trunk theories are the atoms in the partially ordered set that the hierarchy forms. By the way we defined similarity, the different trunk theories of a closed hierarchy each axiomatize a single (atomic) ontological design choice that extends the root theory. For example, the theory of dense partial orderings and the theory of discrete partial orderings form trunk theories for the hierarchy associated with partial orderings. The former introduces the ontological design choice "density" while the latter introduced the ontological design choice of "discreteness". This allows a more formal definition of the informal concept of ontological design choices: Any remainder between a trunk theory and the 
root theory in a hierarchy of the repository axiomatizes the atomic design choice made by a particular trunk theory.

We can now verify that the similarity between trunk theories in the same closed hierarchy is the unique root theory.

Lemma 4 The similarity between any two trunk theories of the same closed hierarchy is logically equivalent to the root theory.

We can introduce an additional property for hierarchies which enforces the condition that every theory in the hierarchy makes a set of atomic design choices. The hierarchy is thereby required to be atomistic, i.e. each theory is the join of a set of atoms (trunk theories).

Definition 11 A closed hierarchy $\mathbb{H}=\langle\mathcal{H}, \leq\rangle$ is atomistic iff every $T \in \mathcal{H}$ that is not a trunk theory satisfies the following property: if $T_{1}, \ldots, T_{n} \in \mathcal{H}$ are all the theories covered by $T$, then $T$ is logically equivalent to $T_{1} \cup \ldots \cup T_{n}$.

Note that this allows for multiple logically equivalent axiomatizations, that is, the set of theories covered by $T$ is not necessarily the minimal set of theories whose union is logically equivalent to $T$. We can define a minimal set by only including trunk theories:

Definition 12 A set of trunk theories $T_{1}, \ldots, T_{n}$ is a profile of a theory $T$ iff $T$ is logically equivalent to $T_{1} \cup \ldots \cup T_{n}$.

We obtain the following theorem similar to what is known about atomistic lattices:

Theorem 1 A non-root theory $T$ in a closed atomistic hierarchy has a unique profile up to logical equivalence.

Proof: Proof by induction over the distance in the partial order $<$ from a trunk theory within the hierarchy $\mathbb{H}=\langle\mathcal{H}, \leq\rangle$.

Base case: Each trunk theory $T \in \mathcal{H}$ has itself as its unique profile, since no other trunk theory is entailed by or entails $T$ (Lemma 4).

Induction step: Assume that $T_{1}, \ldots, T_{n}$ have unique profiles, and $T$ covers a subset thereof, that is, $T$ covers all of $T_{1}, \ldots, T_{k}$ with $k \leq n$. Then $T$ is by the definition of an atomistic hierarchy logically equivalent to the union of the profiles of $T_{1}, \ldots, T_{k}$, which in turn by the definition of profile is the unique profile of $T$.

Consequently every theory in $\mathcal{H}$ has a unique profile.

For example, the ordering hierarchy is atomistic. E.g., the set of three trunk theories $T_{\text {nofinal }}, T_{\text {noinitial }}$, and $T_{\text {linear_ordering }}$, make up the profile of $T_{\text {inf_ordering }}$. Since $T_{n o f i n a l}$ is the theory of partial orders without a final point, $T_{\text {noinitial }}$ is the theory of partial orders without an initial point, and $T_{\text {linear_ordering }}$ is the theory of linear orders, it is easy to see that their union is logically equivalent to the theory of infinite linear orders, $T_{\text {linear_ordering }}$, and thus the three theories indeed form the profile of $T_{\text {linear_ordering }}$. This example illustrates how the profile modularizes a larger theory in the hierarchy into a set of atomic theories. 
Theorem 2 Let $\mathbb{H}=\langle\mathcal{H}, \leq\rangle$ be a closed atomistic hierarchy.

The similarity $S$ of two theories $T_{1}, T_{2} \in \mathcal{H}$ is logically equivalent to the union of the trunk theories that are the intersection of the profile of $T_{1}$ and the profile of $T_{2}$.

Proof: Since the hierarchy is closed, the similarity $S$ of $T_{1}$ and $T_{2}$ is in the hierarchy. Moreover, the hierarchy is atomistic and thus by Thm. $2 S$ has a unique profile in $\mathcal{H}$. Then it is sufficient to prove that the profile of the similarity is equivalent to the intersection of the profiles of $T_{1}$ and $T_{2}$. We show that profile $\left(T_{1}\right) \cap$ $\operatorname{profile}\left(T_{2}\right) \supseteq \operatorname{profile}(S)$ and profile $\left(T_{1}\right) \cap \operatorname{profile}\left(T_{2}\right) \subseteq \operatorname{profile}(S)$. profile $\left(T_{1}\right) \cap \operatorname{profile}\left(T_{2}\right) \supseteq$ profile $(S)$ : Suppose some trunk theory $T$ is in the profile of $S$, then both $T_{1} \models T$ and $T_{2} \models T$. Since by Lemma 4 the similarity of any two trunk theories is the root theory, no other trunk theory can entail $T$. But for $T_{1}$ and $T_{2}$ to be equivalent to their respective profiles, each of the profiles of $T_{1}$ and $T_{2}$ must entail $T$. Hence $T$ is in the profiles of both $T_{1}$ and $T_{2}$, and thus in the intersection of their profiles.

profile $\left(T_{1}\right) \cap$ profile $\left(T_{2}\right) \subseteq$ profile $(S)$ : Since the hierarchy is closed, the similarity $S$ between $T_{1}$ and $T_{2}$ is also in $\mathcal{H}$. Since the hierarchy is atomistic, by Thm. $1 S$ has a unique profile in $\mathcal{H}$. Now it is easy to see that any trunk theory that is both in the profiles of $T_{1}$ and $T_{2}$, must also be in the profile of $S$.

In the previous example, the profile of $T_{\text {dense_linear }}$ is the set of trunk theories of $T_{\text {dense_partial }}$ and of $T_{\text {linear_ordering }}$, and the profile of $T_{\text {discrete_linear }}$ is the set of trunk theories $T_{\text {discrete_partial }}$ and $T_{\text {linear_ordering. }}$. We recall that the similarity of these two theories is a theory of linearly ordered points that makes no commitment with respect to density or discreteness, $T_{\text {linear_ordering }}$, which is equivalent to the intersection of their profiles.

\subsection{Hierarchies and Interoperability}

Ontologies often play a key role in ensuring interoperability between software systems. We can talk about the interoperability of two software systems in terms of the relationships of their underlying ontologies. We say that two software systems can be semantically integrated if the sets of intended models of their (explicit or implicit) ontologies are equivalent. However, systems cannot exchange the models of their ontologies themselves - they can only exchange sentences in the formal language (with a given signature) that they use to represent their knowledge. Suppose that the software systems use as their underlying ontologies two different theories within the same closed hierarchy. How can we use the relationships among the theories in the repository to articulate the nature of interoperability between the software systems relying on those theories?

If the theories of two software systems are logically equivalent, then obviously the two software systems can exchange arbitrary sentences and preserve theorems. If the two theories are not logically equivalent, then their similarity is the shared subtheory that characterizes a set of theorems that can be shared. But how can we capture the sentences that are not in the similarity but are entailed by one of the theories, that is, the sentences that are not shared? If these sentences are mutually inconsistent, then they capture the obstacles to semantic interoperability between the software system. On the other hand, if these sentences that are different among the two theories are mutually consistent, then it is possible for one or the other system to consider extending its theory in order to share 
a larger subtheory. In the remainder of this section, we will use the notion of similarity among theories in a hierarchy to explore a variety of metatheoretic relations between theories through which we can compare theories in such a way as to support shareabiity and reusability.

\subsubsection{Remainders}

The first metatheoretic relation we consider is between a theory $T_{1}$ and one of its nonconservative extensions $T_{2}$.

Definition 13 Let $T_{1}, T_{2}$ be theories in the same hierarchy such that $T_{1}<T_{2}$.

The remainder between $T_{2}$ and $T_{1}$ is the weakest theory (up to logical equivalence) $T^{\prime}$ with $\Sigma\left(T^{\prime}\right)=\Sigma\left(T_{1}\right)$ so that

$$
T_{2} \equiv T_{1} \cup T^{\prime} .
$$

Intuitively, the remainder constitutes the weakest axiomatization that suffices to extend $T_{1}$ to $T_{2}$; in particular it must contain the ontological design choices of $T_{2}$ that are not contained in $T_{1}$. As such, this set of axioms may not be itself in the hierarchy or the entire repository. In fact, the next lemma shows that remainders do not extend the common root theory in a closed hierarchy and are therefore not themselves in the hierarchy if the hierarchy is closed.

Lemma 5 Let $T_{1}, T_{2}$ be theories in the same closed hierarchy with $T_{1}<T_{2}$. The remainder between $T_{2}$ and $T_{1}$ is non-empty and is not in the closed hierarchy.

Proof: Since $T_{1}<T_{2}$, that is, $\operatorname{Mod}\left(T_{2}\right) \subsetneq \operatorname{Mod}\left(T_{1}\right)$, a sentence $\sigma$ capturing a model in $\operatorname{Mod}\left(T_{1}\right) \backslash \operatorname{Mod}\left(T_{2}\right)$ is not consistent with $T_{2}$, that is $T_{2} \models \neg \sigma$, but is consistent with $T_{1}$, that is, $T_{1} \not \models \neg \sigma$. Then by the definition of remainder, $T^{\prime} \models \neg \sigma$, therefore $T^{\prime}$ is non-empty.

The closed hierarchy has a unique root $T^{\text {root }}$ by Lemma 3. Suppose $T^{\prime}$ is in the hierarchy, then it must extend $T^{r o o t}$. But then, by the definition of the remainder, there exists a theory $T^{\prime \prime}$ that is weaker than $T^{\prime}$ which does not entail $T^{r o o t}$. Then $T^{\prime}$ is not the remainder.

Consider the theories of linearly ordered points, $T_{\text {linear_ordering, }}$, and of partially ordered points, $T_{\text {partial_ordering }}$. The minimal set of axioms required to extend $T_{\text {partial_ordering }}$ to $T_{\text {linear_ordering }}$ is the axiom expressing that the order is total and as such, that axiom constitutes the remainder. However, that single axiom alone does not extend the root theory and therefore is not in the closed hierarchy of ordering theories. 


\subsubsection{Differences}

Though remainders are not included in closed hierarchies, they lend themselves to a definition of differences between a theory and a non-conservative extension thereof.

Definition 14 Let $T_{1}, T_{2}$ be theories in the same closed hierarchy $\mathbb{H}$ so that $T_{1}<T_{2}$. Let $T^{\prime}$ be the remainder between $T_{2}$ and $T_{1}$ and let $T^{\text {root }}$ be the unique root theory of $\mathbb{H}$.

The difference between $T_{2}$ and $T_{1}$ is the theory (up to logical equivalence) $T^{d}$ with $\Sigma\left(T^{d}\right)=\overline{\Sigma\left(T_{1}\right) \text { so that }}$

$$
T^{d} \equiv T^{r o o t} \cup T^{\prime}
$$

For example, the difference of $T_{\text {dense_linear }}$ and $T_{\text {linear_ordering }}$ in the ordering hierarchy is the theory that extends the root theory with the remainder between $T_{\text {dense_linear }}$ and $T_{\text {linear_ordering. }}$. The remainder between those two theories is the axiom that enforces density between points; extending the root theory $T_{\text {partial_ordering }}$ with this remainder yields the difference $T_{\text {dense_partial }}$.

It is easy to see that $T^{d}$ non-conservatively extends $T^{\text {root }}$ and that $T_{2}$ nonconservatively extends $T^{d}$. Moreover, non-empty differences always exist by Def. 14 .

Even though differences may not be included in a hierarchy, they are always compatible with the hierarchy. In closed atomistic hierarchies we can easily obtain the difference between any two constituent theories from the trunk theories of the hierarchy as follows.

Theorem 3 Let $T_{1}, T_{2}$ be theories in the same closed atomistic hierarchy with $T_{1}<T_{2}$. The difference $T^{d}$ between $T_{2}$ and $T_{1}$ is logically equivalent to the set difference of the profile of $T_{2}$ and the profile of $T_{1}$.

Proof: Let the trunk theories $T_{21}, \ldots, T_{2 n}$ be the profile of $T_{2}$, that is $T_{2} \equiv T_{21} \cup \ldots \cup T_{2 n}$. The profiles of $T_{1}$ and $T^{d}$ are then subsets of the profile of $T_{2}$. Suppose some $T_{2 i}$ is in the profiles of $T_{1}$ and $T^{d}$. Then $T_{2 i}$ is in the similarity of $T_{1}$ and $T^{d}$ and by Lemma $3, T^{\text {root }} \models T_{2 i}$, so $T_{2 i}$ is not a trunk theory - a contradiction. Thus the profiles are disjoint subsets of profile $\left(T_{2}\right)$.

Now suppose there is a trunk theory $T_{2 i}$ in the profile of $T_{2}$ that is neither in the profile of $T_{1}$ nor of $T^{d}$, that is $T_{1} \not \models T_{2 i}$ and $T^{d} \not \models T_{2 i}$. With $T_{2} \models T_{2 i}$, this violates the definition of difference (with the definition of remainder).

For example, the profile of $T_{\text {dense_linear }}$ is the set of trunk theories $T_{\text {dense_partial }}$ and $T_{\text {linear_ordering }}$, and the profile of trunk theory $T_{\text {linear_ordering }}$ is itself. As described in an earlier example, the difference between $T_{\text {dense_linear }}$ and $T_{\text {linear_ordering }}$ is the theory that extends the root theory with the axiom enforcing density between points, $T_{\text {dense_partial }}$. Comparing the set difference of the profiles of $T_{\text {dense_partial }}$ and $T_{\text {linear_ordering }}$, we see that it is equivalent to the difference between those theories. 


\section{Relationships between Hierarchies}

Theories within the same hierarchy have the same non-logical lexicon; if we wish to consider the different relationships between theories in different hierarchies, we consider two cases - either the non-logical lexicon of one theory is an expansion of the non-logical lexicon of the other theory $\left(\Sigma\left(T_{i}\right) \subset \Sigma\left(T_{j}\right)\right)$ or the non-logical lexicons of the two theories are disjoint $\left(\Sigma\left(T_{i}\right) \cap \Sigma\left(T_{j}\right)=\emptyset\right)$. In this section, we explore these two possibilities and discuss their roles in providing an adequate notion of ontology modularity.

\subsection{Hierarchies and Conservative Extensions}

The simplest relationship between theories that are in different hierarchies is that of extension. In particular, the notion of conservative extension (see Definition 3) has played a key role in the study of modular ontologies $[18,9]$.

The following theorem shows that we do not speak of one hierarchy $\mathbb{H}_{1}$ being a nonconservative extension of another hierarchy $\mathbb{H}_{2}$, since we can always add new theories to $\mathbb{H}_{2}$ that are conservatively extended by theories in $\mathbb{H}_{1}$.

Theorem 4 Suppose $T_{1}, T_{2}$ are theories that are in different hierarchies such that $\Sigma\left(T_{1}\right) \subset \Sigma\left(T_{2}\right)$.

If $T_{2}$ is a non-conservative extension of $T_{1}$, then there exists a theory $T_{3}$ such that

- $T_{2}$ is a conservative extension of $T_{3}$, and

- $T_{3}$ is compatible with the hierarchy of $T_{1}$, i.e., $\Sigma\left(T_{3}\right)=\Sigma\left(T_{1}\right)$.

Proof: Suppose $T_{2}$ is a non-conservative extension of $T_{1}$ and $\Sigma\left(T_{1}\right) \subset \Sigma\left(T_{2}\right)$.

If

$$
\Omega=\left\{\omega: \omega \in \mathcal{L}\left(T_{1}\right), T_{1} \not \models \omega, T_{2} \models \omega\right\}
$$

then $T_{3}=T_{1} \cup \Omega$ is a non-conservative extension of $T_{1}$ with $\Sigma\left(T_{1}\right)=\Sigma\left(T_{3}\right)$, and $T_{2}$ is a conservative extension of $T_{1} \cup \Omega$.

If we include the new theory $T_{3}$ in the hierarchy containing $T_{1}$, the non-conservative extensions is limited to this hierarchy.

An example of a modular ontology that is organized through both conservative and non-conservative extensions is the PSL Ontology [12]. The theories that constitute PSL Outer Core are shown in Figure $3^{5}$. The theory $T_{\text {occtree }}$ is a non-conservative extension of $T_{\text {pslcore }}$ which also expands the non-logical lexicon. Following Theorem 4, there exists a theory $T_{a o a}$ that is a non-conservative extension of $T_{p s l c o r e}$ within the same hierarchy such that $T_{\text {occtree }}$ is a conservative extension of $T_{a o a}$. Similarly, the theory $T_{\text {atomic }}$ is a non-conservative extension of $T_{\text {subactivity }}$ which also expands the non-logical lexicon. The theory $T_{\text {sublat }}$ is a non-conservative extension of $T_{\text {subactivity }}$ within the same hierarchy such that $T_{\text {atomic }}$ is a conservative extension of $T_{\text {sublat }}$.

In the case of using $\mathcal{E}$-connections to decompose a large ontology into modules (a collection of axioms), each module encapsulates some terms of the original ontology.

\footnotetext{
${ }^{5}$ The CLIF axiomatization of these theories can be found at http://stl.mie.utoronto.ca/colore/process/
} 


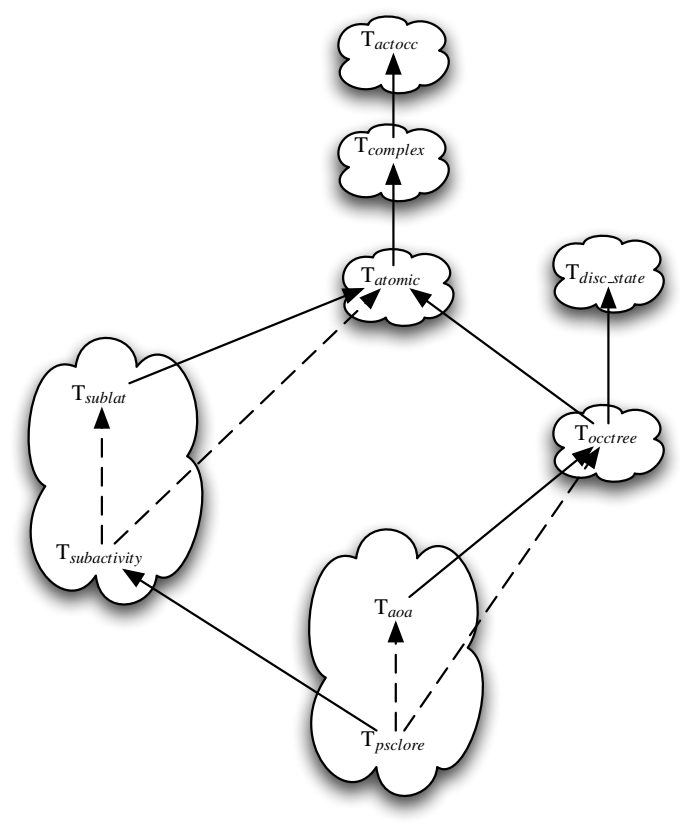

Figure 3. Relationships between the theories in PSL Outer Core. Clouds indicate different hierarchies. Dashed lines denote non-conservative extension while solid lines denote conservative extensions.

In [8] the definition of semantic encapsulation is given as a component that preserves a basic set of entailments of a term in an ontology. This leads to the partitioning of a large ontology into a collection of modules that are conservative extensions of one another. The relationship of conservative extensions between modules ensures that each module can be reused independent of the rest while retaining the original semantics of its contained terms. The major limitation of using $\mathcal{E}$-connections is that the use of such modules as a means of refining an ontology (non-conservative extensions of a theory) becomes impossible.

Using only the relationship of conservative extension does not give us a sufficient characterization of the granularity of a module. PSL Outer Core gives us examples of theories that are conservative extensions, yet we can decompose them into smaller theories through reducibility. Furthermore, there are also conservative extensions that are irrelevant to modularity. For example, the Peano Arithmetic is a conservative extension of the theory of linear orderings, yet we would not say that the latter theory forms a module within the former theory.

\subsection{Interpretability between Theories}

Ontologies whose non-logical lexicons are expansions of other non-logical lexicons can be compared using the notions of satisfiability, extension, and independence. More difficult is to compare ontologies that are axiomatized using different non-logical lexicons; in such cases, we need to determine whether or not the non-logical lexicon of one ontology can be interpreted in the non-logical lexicon of the other ontology. For this purpose 
we assume that the two theories use disjoint non-logical lexicon; if they are not disjoint a simple renaming of one of the lexicons will do the trick. In this section, we consider metatheoretic relationships between theories based on the notion of relative interpretation, and we also discuss how they can support an approach to ontology modularity through the organization of ontologies into a repository.

\subsubsection{Relative Interpretation}

The notion of interpretability between theories is widely used within mathematical logic and applications of ontologies for semantic integration ([22]). We will adopt the following definition from [5]:

Definition 15 An interpretation $\pi$ of the theory $T_{1}$ with signature $\Sigma\left(T_{1}\right)$ into a theory

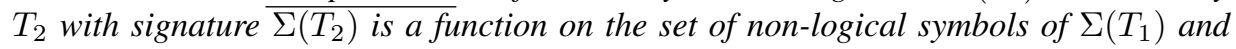
formulae in $\mathcal{L}\left(T_{1}\right)$ such that

1. $\pi$ assigns to $\forall$ a formula $\pi_{\forall}$ of $\mathcal{L}\left(T_{2}\right)$ in which at most the variable $v_{1}$ occurs free, such that

$$
T_{2} \models\left(\exists v_{1}\right) \pi_{\forall}
$$

2. $\pi$ assigns to each n-place relation symbol $P$ a formula $\pi_{P}$ of $\mathcal{L}\left(T_{2}\right)$ in which at most the variables $v_{1}, \ldots, v_{n}$ occur free.

3. $\pi$ assigns to each n-place function symbol $f$ a formula $\pi_{f}$ of $\mathcal{L}\left(T_{1}\right)$ in which at most the variables $v_{1}, \ldots, v_{n}, v_{n+1}$ occur free, such that

$$
\begin{gathered}
T_{1} \models\left(\forall v_{1}, \ldots, v_{n}\right) \pi_{\forall}\left(v_{1}\right) \wedge \ldots \wedge \pi_{\forall}\left(v_{n}\right) \\
\supset(\exists x)\left(\pi_{\forall}(x) \wedge\left(\left(\forall v_{n+1}\right)\left(\pi_{f}\left(v_{1}, \ldots, v_{n+1}\right) \equiv\left(v_{n+1}=x\right)\right)\right)\right)
\end{gathered}
$$

4. for any atomic sentence $\sigma \in \mathcal{L}\left(T_{1}\right)$ with relation symbol $P, \pi(\sigma)=\pi(P)$;

5. for any sentence $\sigma \in \mathcal{L}\left(T_{1}\right)$,

$$
\pi(\neg \sigma)=\neg \pi(\sigma) \text {; }
$$

6. for any sentences $\sigma, \tau \in \mathcal{L}\left(T_{1}\right)$, $\pi(\sigma \rightarrow \tau)=\pi(\sigma) \rightarrow \pi(\tau)$

7. for any sentence $\sigma \in \mathcal{L}\left(T_{1}\right)$, $\pi(\forall x \sigma)=\forall x \pi_{\forall} \rightarrow \pi(\sigma)$;

8. For any sentence $\sigma \in \mathcal{L}\left(T_{1}\right)$,

$$
T_{1} \models \sigma \Rightarrow T_{2} \models \pi(\sigma)
$$

Thus, the mapping $\pi$ is an interpretation of $T_{1}$ if it preserves the theorems of $T_{1}$ and we say $T_{1}$ is interpretable in $T_{2}$.

Definition 16 An interpretation $\pi$ of a theory $T_{1}$ into a theory $T_{2}$ is faithful iff

$$
T_{1} \not \models \sigma \Rightarrow T_{2} \not \models \pi(\sigma)
$$

for any sentence $\sigma \in \mathcal{L}\left(T_{1}\right)$. 
Thus, the mapping $\pi$ is a faithful interpretation of $T_{1}$ if it preserves satisfiability with respect to $T_{1}$. We will also refer to this by saying that $T_{1}$ is faithfully interpretable in $T_{2}$. For example, the work in [11] shows that the PSL-Core theory within the PSL Ontology [12] is interpretable by Reiter's axiomatization of situation calculus, but that this is not a faithful interpretation, since there are sentences consistent with PSL-Core that are not consistent with situation calculus.

Definable equivalence is a generalization of the notion of logical equivalence to theories that do not have the same signature.

Definition 17 Two theories $T_{1}$ and $T_{2}$ are definably equivalent iff $T_{1}$ is faithfully interpretable in $T_{2}$ and $T_{2}$ is faithfully interpretable in $T_{1}$.

For example, the theory of timepoints is definably equivalent to the theory of linear orderings. On the other hand, although the theory of partial orderings is interpretable in the theory of timepoints, these two theories are not definably equivalent, since the theory of timepoints is not interpretable in the theory of partial orderings.

Faithful interpretations are a generalization of the notion of conservative extension, leading to the following generalization of Theorem 4:

Theorem $5 T_{1}$ is faithfully interpretable in $T_{2}$ iff there is theory $T_{3}$ such that $T_{1}$ is definably equivalent to $T_{3}$ and $T_{2}$ is a conservative extension of $T_{3}$.

Proof: Suppose $T_{1}$ is definably equivalent to $T_{3}$ and $T_{2}$ is a conservative extension of $T_{3}$.

By the definition of definable equivalence, we know that $T_{1}$ is faithfully interpretable in $T_{3}$, so that there exists an interpretation $\pi$ of $T_{1}$ into $T_{3}$ such that

$$
T_{1} \models \sigma \Leftrightarrow T_{3} \models \pi(\sigma)
$$

Since $T_{2}$ is a conservative extension of $T_{3}$, we have

$$
T_{3} \models \sigma \Leftrightarrow T_{2} \models \pi(\sigma)
$$

and hence $T_{1}$ is faithfully interpretable in $T_{2}$.

For the converse, suppose that $T_{1}$ is faithfully interpretable in $T_{2}$ with the interpretation $\pi$. Let

$$
T_{3}=\left\{\pi(\sigma): \sigma \in T_{1}\right\}
$$

Note that $\Sigma\left(T_{3}\right)=\Sigma\left(T_{1}\right)$, and that $T_{3}$ is definably equivalent to $T_{1}$.

$T_{2}$ is a conservative extension of $T_{3}$; otherwise, there exists a sentence $\sigma \in \mathcal{L}\left(T_{3}\right)$ such that

$$
T_{3} \not \models \sigma \quad \text { and } \quad T_{2} \models \sigma
$$

However, since $T_{3}$ is definably equivalent to $T_{1}$, this would mean that

$$
T_{1} \not \models \sigma
$$

which contradicts the definable equivalence of $T_{1}$ and $T_{2}$. 
The use of relative interpretations between first-order axiomatized theories as a means to explicitly combine smaller theories was introduced by [1]. This approach has been implemented by the Interactive Mathematical Proof System (IMPS) [6], which is theorem prover that utilizes a repository of axiomatized mathematical theories linked to each other through relative interpretations using the little theories approach to mechanize traditional tools of classical mathematical reasoning [7]. The use of relative interpretations by IMPS provide the means to transport a theorem from the theory it was proved into any other theory linked with an interpretation. The IMPS repository is organized around the relative interpretations available between stored theories. Furthermore, IMPS guarantees the consistency of generated proofs based on the notion of relative consistency between theories. Within IMPS there is a set of theories deemed foundational, meaning they are regarded or known to be consistent. Since all proofs begin with a foundational theory and any theory developed from another is a conservative extension of the original theory, all theories developed are consistent relative to the original foundational theory [6]. Although the use and definitions of theory interpretations and relative consistency in IMPS are specific to the purpose of theorem proving, it nonetheless shows how such relationships can be utilized to relate and combine theories.

Nevertheless, since relative interpretation and definable equivalence are generalizations of the notion of extension, these relationships between theories suffer from the same drawbacks when attempting to decompose an ontology into modules. In particular, knowing that one theory $T_{1}$ is interpretable by a theory $T_{2}$ does not explicitly identify the subtheory of $T_{1}$ that is definably equivalent to $T_{2}$.

\subsubsection{Reducibility}

Definable equivalence is a relationship between two theories; we can generalize this to a relationship among a set of theories. The basis for this approach is the model-theoretic notion of reducibility introduced in [13]. In this paper, we characterize the relationship between theories that corresponds to the model-theoretic notion of reducibility.

Definition 18 theory $T$ is reducible to a set of theories $T_{1}, \ldots, T_{n}$ iff

1. $T$ faithfully interprets each theory $T_{i}$, and

2. $T_{1} \cup \ldots \cup T_{n}$ faithfully interprets $T$.

We will also refer to the set of theories $T_{1}, \ldots, T_{n}$ in the definition as the reduction of $T$ in the repository.

It is easy to see that two definably equivalent theories are reducible to each other. For example, the theory of timepoints is reducible to the theory of linear ordering and vice versa.

A nontrivial example of reducibility can be seen with $T_{\text {pslcore }}$ within the PSL Ontology (see Figure 4). As discussed in [13], $T_{p s l c o r e}$ is reducible to $T_{\text {linear }}, T_{\text {partition }}$, $T_{\text {graph-incidence. }}$. This example also illustrates how the notion of reducibility leads to the decomposition of a theory that is treated as a module within a larger ontology, addressing the issue of the granularity of modules. 


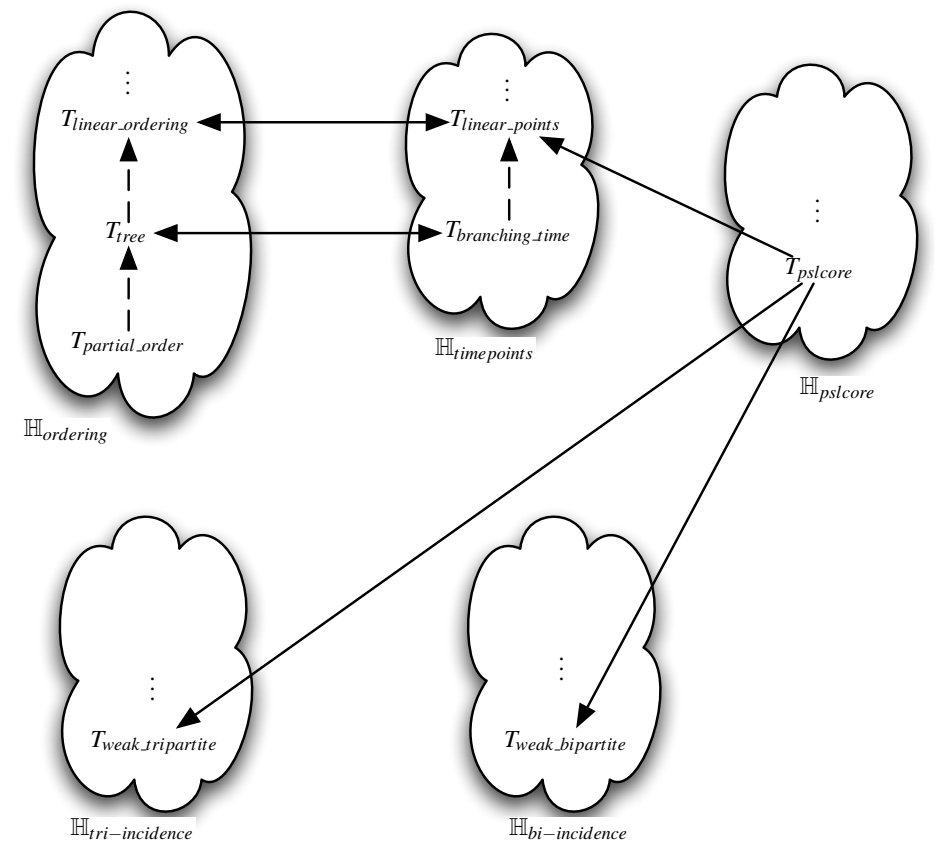

Figure 4. A depiction of the reducibility of $T_{\text {pslcore }}$ to other core theories. Clouds indicate hierarchies; only the root theory of each hierarchy and other relevant theories are shown. Dashed lines denote non-conservative extension within a hierarchy and solid lines denote reducibility of theories between hierarchies.

Lemma 6 Let $T_{1}, \ldots, T_{n}$ be a set of theories such that $\Sigma\left(T_{i}\right) \cap \Sigma\left(T_{j}\right)=\emptyset$ for all $1 \leq$ $i, j \leq n, i \neq j$.

If $T$ faithfully interprets each $T_{1}, \ldots, T_{n}$, then $T$ faithfully interprets $T_{1} \cup \ldots \cup T_{n}$.

Proof: Since the signatures of the theories are disjoint, we can construct an interpretation $\pi$ of $T_{1} \cup \ldots \cup T_{n}$ in $T$ by the union of the interpretations $\pi_{i}$ of each $T_{i}$ in $T$.

Theorem 6 Let $T_{1}, \ldots, T_{n}$ be a set of theories such that $\Sigma\left(T_{i}\right) \cap \Sigma\left(T_{j}\right)=\emptyset$ for all $1 \leq i, j \leq n, i \neq j$.

A theory $T$ is reducible to $T_{1}, \ldots, T_{n}$ iff $T$ is definably equivalent to

$$
T_{1} \cup \ldots \cup T_{n}
$$

Proof: Let $T_{1}, \ldots, T_{n}$ be a set of theories such that $\Sigma\left(T_{i}\right) \cap \Sigma\left(T_{j}\right)=\emptyset$ for all $1 \leq i, j \leq$ $n, i \neq j$.

If $T$ is reducible to $T_{1}, \ldots, T_{n}$, then $T_{1} \cup \ldots \cup T_{n}$ (faithfully) interprets $T$; by Lemma $6 T$ (faithfully) interprets $T_{1} \cup \ldots \cup T_{n}$, so it is easy to see that $T$ is definably equivalent to $T_{1} \cup \ldots \cup T_{n}$. 
Suppose $T$ is not reducible to a set of theories $T_{1}, \ldots, T_{n}$ but $T$ is definably equivalent to $T_{1} \cup \ldots \cup T_{n}$. Either $T$ does not faithfully interpret some $T_{i}$ or $T_{1} \cup \ldots \cup T_{n}$ does not faithfully interpret $T$. In the first case, there exists a sentence $\sigma \in \mathcal{L}\left(T_{i}\right)$ and an interpretation $\pi$ such that

$$
T_{i} \not \models \sigma, T \models \pi(\sigma)
$$

However, $T$ is definably equivalent to $T_{1} \cup \ldots \cup T_{n}$, so that any sentence entailed by $T$ is also entailed by $T_{1} \cup \ldots \cup T_{n}$, and since $\sigma \in \mathcal{L}\left(T_{i}\right)$, we must have $T_{i} \models$ $\sigma$, which violates the assumption that $T$ does not faithfully interpret some $T_{i}$. A similar argument applies for the other case, thus contradicting the assumption that $T$ is not reducible to a set of theories $T_{1}, \ldots, T_{n}$.

Corollary 1 If $T_{1}$ is definably equivalent to $T_{2}$, then $T_{1}$ is reducible to $T_{2}$.

In the following, we will see that reducibility is the metatheoretic relation we need for both the organization of ontology repositories and a definition of modules within an ontology.

\subsection{Ordering Hierarchies by Faithful Interpretation}

In the same way that we defined a hierarchy to be a set of theories that are partially ordered by extension, we can use faithful interpretation and reducibility to define a quasiordering over hierarchies.

Definition 19 Let $\mathbb{H}_{i}=\left\langle\mathcal{H}_{i}, \leq\right\rangle$ and $\mathbb{H}_{j}=\left\langle\mathcal{H}_{j}, \leq\right\rangle$ be hierarchies.

$\mathbb{H}_{i} \preceq \mathbb{H}_{j}$ iff each root theory in $\mathcal{H}_{j}$ faithfully interprets a theory $T$ that is compatible with $\mathcal{H}_{i}$.

For example ${ }^{6}$, we have the following relationships:

$$
\mathbb{H}_{\text {between }} \preceq \mathbb{H}_{\text {ordering }} \preceq \mathbb{H}_{\text {timepoints }} \preceq \mathbb{H}_{\text {pslcore }}
$$

Since the $\preceq$ relation on hierarchies plays a key role, we show that it is indeed an ordering relation on the set of hierarchies.

Lemma 7 If $T_{1}$ is faithfully interpretable in $T_{2}$ and $T_{2}$ is faithfully interpretable in $T_{3}$, then $T_{1}$ is faithfully interpretable in $T_{3}$.

Proof: $T_{1}$ is faithfully interpretable in $T_{2}$ iff there exists an interpretation $\pi_{1}$ such that

$$
T_{1} \models \sigma \Leftrightarrow T_{2} \models \pi_{1}(\sigma)
$$

\footnotetext{
${ }^{6} \mathrm{We}$ adopt a naming convention for hierarchies similar to the one that we use for theories. Each hierarchy is associated with a URI such that the subscript in the hierarchy name is the unqualified name in the URI. For example, the theories in the hierarchy $\mathbb{H}_{\text {ordering }}$ can be found at http://stl.mie.utoronto.ca/colore/ordering/.

Also note that the hierarchy $\mathbb{H}_{\text {ordering }}$ is depicted in Figure 1 and that the hierarchy $\mathbb{H}_{\text {timepoints }}$ is depicted in Figure 2.
} 
$T_{2}$ is faithfully interpretable in $T_{3}$ iff there exists an interpretation $\pi_{2}$ such that

$$
T_{2} \models \sigma \Leftrightarrow T_{3} \models \pi_{2}(\sigma)
$$

Combining these gives us

$$
T_{1} \models \sigma \Leftrightarrow T_{3} \models \pi_{2} \pi_{1}(\sigma)
$$

so that $\pi_{2} \pi_{1}$ is a faithful interpretation of $T_{1}$ in $T_{1}$.

Lemma 8 If $T_{1}$ is faithfully interpretable in $T_{2}$ and $T_{1}<T_{3}$, then $T_{3}$ is faithfully interpretable in some extension of $T_{2}$.

Proof: $T_{1}$ is faithfully interpretable in $T_{2}$ iff there is an interpretation $\pi$ such that

$$
T_{1} \models \omega \Leftrightarrow T_{2} \models \pi(\omega)
$$

Suppose $T_{1}<T_{3}$ (so that $T_{1}$ and $T_{3}$ are in the same hierarchy) and let

$$
\begin{gathered}
\Omega=\left\{\omega: T_{3} \models \omega, T_{1} \not \models \omega\right\} \\
\pi(\Omega)=\{\pi(\omega): \omega \in \Omega\}
\end{gathered}
$$

We can specify a new theory

$$
T_{4}=T_{2} \cup \pi(\Omega)
$$

such that

$$
T_{3} \models \omega \Leftrightarrow T_{4} \models \pi(\omega)
$$

so that $T_{3}$ is faithfully interpretable in an extension of $T_{2}$.

Lemma 9 The $\preceq$ relation is a quasiordering on the set of hierarchies.

Proof: $\preceq$ is reflexive because the root theory of any hierarchy is reducible to itself.

Suppose

$$
\mathbb{H}_{1} \preceq \mathbb{H}_{2}, \mathbb{H}_{2} \preceq \mathbb{H}_{3}
$$

Each root theory $T_{3 i}^{\text {root }}$ faithfully interprets some theory $T_{2 i}$ that is compatible with $\mathbb{H}_{2}$.

There exists a root theory in $\mathbb{H}_{2}$ such that $T_{2 i}^{r o o t}<T_{2 i}$ and this root theory faithfully interprets some theory $T_{1 i}$ compatible with $\mathbb{H}_{1}$.

By Lemma 8, $T_{2 i}$ faithfully interprets an extension $T_{11}$ of $T_{2 i}$.

By Lemma 7, the root theory $T_{3 i}^{\text {root }}$ faithfully interprets some theory $T_{11}$ compatible with $\mathbb{H}_{1}$, and hence $\mathbb{H}_{1} \preceq \mathbb{H}_{3}$. 
From the example presented at the beginning of this section, we can already see that this ordering relation alone will not be sufficient as the basis for an approach to modularity. The theories of betweenness relations in $\mathbb{H}_{\text {between }}$ are intuitively the weakest, since they can be faithfully interpreted in the other theories in the example. However, theories in $\mathbb{H}_{\text {ordering }}$ are not reducible to theories in $\mathbb{H}_{\text {between }}$. As a result, models of the theories in $\mathbb{H}_{\text {between }}$ cannot be used to construct models of the theories in the other hierarchies such as orderings, so that the theories in $\mathbb{H}_{\text {between }}$ fail to meet the intuition that the weakest theories form modules that are the building blocks of other theories.

\subsection{What is an Ontology Repository?}

In this section, we investigate the use of reducibility as a means of organizing the hierarchies in a repository. Recall that even though an actual repository can only contain ontologies which are concrete sets of axioms, we will again discuss the more abstract repository of theories.

Definition 20 Let $\mathbb{H}_{1}, \ldots, \mathbb{H}_{n}$ be a finite set of hierarchies.

A repository $\mathbb{R}=\langle\mathcal{R}, \sqsubseteq\rangle$ is a partially ordered set where

- $\mathcal{R}=\left\{\mathbb{H}_{1}, \ldots, \mathbb{H}_{n}\right\}$;

- $\mathbb{H}_{i} \sqsubseteq \mathbb{H}_{j}$ iff each root theory in $\mathbb{H}_{j}$ has a reduction that contains a theory $T$ that is compatible with $\mathbb{H}_{i}$.

For example, we can see that $\mathbb{H}_{\text {ordering }} \sqsubseteq \mathbb{H}_{\text {timepoints }}$, since the root theory in $\mathbb{H}_{\text {timepoints }}$ is definably equivalent to the theory $T_{\text {linear_ordering }}$ in $\mathbb{H}_{\text {ordering. }}$. On the other hand, $\mathbb{H}_{\text {timepoints }} \not \subset \mathbb{H}_{\text {ordering }}$, since the root theory for $\mathbb{H}_{\text {ordering }}$ (which is $T_{\text {partial_ordering }}$ ) is not reducible to any theory in $\mathbb{H}_{\text {timepoints }}$.

The reducibility of $T_{\text {pslcore }}$ leads to the following relationships among hierarchies (which is also depicted in Figure 4):

$$
\begin{gathered}
\mathbb{H}_{\text {ordering }} \sqsubseteq \mathbb{H}_{\text {timepoints }} \sqsubseteq \mathbb{H}_{\text {pslcore }} \\
\mathbb{H}_{\text {tri_incidence }} \sqsubseteq \mathbb{H}_{\text {pslcore }} \\
\mathbb{H}_{\text {bi_incidence }} \sqsubseteq \mathbb{H}_{\text {pslcore }}
\end{gathered}
$$

It is important to note that the $\sqsubseteq$ on hierarchies is distinct from this ordering based on faithful interpretation. For example, theories of betweenness relations are faithfully interpretable in theories of orderings relations, but they are not reducible to theories of orderings. Consequently, the hierarchies containing these theories would be incomparable in the repository with respect to the $\sqsubset$ ordering.

Nevertheless, we do have the following relationships between the two orderings, which follows easily from the definition of reducibility:

Lemma 10 Let $\mathbb{H}_{i}=\left\langle\mathcal{H}_{i}, \leq\right\rangle$ and $\mathbb{H}_{j}=\left\langle\mathcal{H}_{j}, \leq\right\rangle$ be hierarchies.

$$
\mathbb{H}_{i} \sqsubseteq \mathbb{H}_{j} \Rightarrow \mathbb{H}_{i} \preceq \mathbb{H}_{j}
$$


We can use earlier results about the $\preceq$ to establish properties of the $\sqsubseteq$ ordering over hierarchies.

Theorem 7 A repository $\mathbb{R}=\langle\mathcal{R}, \sqsubseteq\rangle$ is a quasiordering on the set of hierarchies.

Proof: $\sqsubseteq$ is reflexive because the root theory of any hierarchy is reducible to itself.

Now suppose $\mathbb{H}_{i} \sqsubseteq \mathbb{H}_{j}$ and $\mathbb{H}_{j} \sqsubseteq \mathbb{H}_{k}$.

By Lemma 10, we know $\mathbb{H}_{i} \preceq \mathbb{H}_{j}$ and $\mathbb{H}_{j} \preceq \mathbb{H}_{k}$.

By Lemma 7, we know $\mathbb{H}_{i} \preceq \mathbb{H}_{k}$.

Thus, each root theory in $\mathbb{H}_{k}$ faithfully interprets a theory compatible with $\mathbb{H}_{i}$.

Since $\mathbb{H}_{j} \sqsubseteq \mathbb{H}_{k}$, there exist theories $S_{1}, \ldots, S_{j}, \ldots, S_{n}$ such that $S_{1} \cup \ldots \cup S_{j} \cup \ldots \cup S_{n}$ faithfully interprets a root theory $S_{k}$ in $\mathbb{H}_{k}$.

Since $\mathbb{H}_{i} \sqsubseteq \mathbb{H}_{j}$, there exist theories $T_{1}, \ldots, T_{i}, \ldots, T_{m}$ such that $T_{1} \cup \ldots \cup T_{i} \cup \ldots \cup T_{m}$ faithfully interprets a root theory $S$ in $\mathbb{H}_{j}$.

By Lemma 8, $T_{1} \cup \ldots \cup T_{i}^{\prime} \cup \ldots \cup T_{m}$ faithfully interprets $S_{j}$ in $\mathbb{H}_{j}$.

By Lemma 7, $S_{1} \cup \ldots \cup\left(T_{1} \cup \ldots \cup T_{i}^{\prime} \cup \ldots \cup T_{m}\right) \cup \ldots \cup S_{n}$ faithfully interprets $S_{k}$.

By Theorem 6, $S_{k}$ is reducible to this set of theories and consequently $\mathbb{H}_{i} \sqsubseteq \mathbb{H}_{j}$.

Thus, $\sqsubseteq$ is transitive.

\subsection{Core Hierarchies}

Since we are dealing with repositories that contain a finite set of hierarchies, we are guaranteed that the partial ordering $\sqsubseteq$ has minimal elements.

Definition 21 A hierarchy $\mathbb{C}=\langle\mathcal{C}, \leq\rangle$ is a core hierarchy iff it is a minimal hierarchy in the repository $\mathbb{R}=\langle\mathcal{R}, \sqsubseteq\rangle$.

A theory $T$ is a core theory iff it is compatible with a core hierarchy.

The core repository is the set of core hierarchies within a repository $\mathbb{R}$.

A complex hierarchy $\mathbb{H}=\langle\mathcal{H}, \leq\rangle$ is a hierarchy which is not minimal in the repository $\langle\mathcal{R}, \sqsubseteq\rangle$.

A theory $T$ is a complex theory iff it is compatible with a complex hierarchy.

Using the examples of the preceding section, $\mathbb{H}_{\text {ordering }}$ is a core hierarchy and all theories that axiomatize different classes of orderings are core theories. On the other hand, $\mathbb{H}_{\text {timepoint }}$ and $\mathbb{H}_{\text {pslcore }}$ are complex hierarchies. In particular, $T_{\text {pslcore }}$ is a complex theory; as we saw in Figure 4, this theory is reducible to a set of core theories. The next theorem shows that this property holds for all complex theories:

Theorem 8 If $\mathbb{H}=\langle\mathcal{H}, \leq\rangle$ is a complex hierarchy, then each theory in $\mathcal{H}$ is reducible to a set of core theories.

Proof: Suppose that $\mathbb{R}=\langle\mathcal{R}, \sqsubseteq\rangle$ is a repository such that $\mathcal{R}=\left\{\mathcal{H}_{1}, \ldots, \mathcal{H}_{n}\right\}$.

Let $\mathbb{H}$ be a complex hierarchy in $\mathcal{R}$, so that it is not minimal in the $\sqsubseteq$ quasiordering (following Theorem 7). Because the repository is finite, and all finite quasiorderings are well-founded, there exist core hierarchies $\mathbb{C}_{1}, \ldots, \mathbb{C}_{n}$ with $\mathbb{C}_{i}=\left\langle\mathcal{C}_{i}, \leq\right\rangle$ such that

$$
\mathbb{C}_{i} \sqsubseteq \mathbb{H}
$$


By the definition of $\sqsubseteq$, each root theory $T$ in $\mathbb{H}$ is reducible to

$$
T_{1}, \ldots, T_{n}
$$

where $T_{i} \in \mathcal{C}_{i}$. Since core hierarchies are minimal in the $\sqsubseteq$ quasiordering, all of these theories $T_{i}$ are core theories.

Theorem 8 shows us that core theories are intuitively the building blocks of complex theories, analogous to the way that trunk theories are the building blocks of other theories in the same hierarchy. These insights lead us to a characterization of modularity.

\subsection{Relationships between Theories}

Thus far in the paper, we have used three different metatheoretic relationships to organize a first-order ontology repository - non-conservative extension (within a hierarchy), faithful interpretation, and reducibility (between different hierarchies). In each case, we can specify a partial ordering over the set of theories.

There have been several other approaches that have used similar relationships. In his lattice of theories, Sowa [23] describes a partial ordering over all first-order theories based on the notion of generalization and specialization. The top element of the lattice is the set of first-order tautologies, and each theory below the top is an extension of the ones above it. Sowa informally proposes four operations between theories within the lattice - contraction, expansion, revision, and analogy. Although he does not propose formal definitions for these operations, it appears that analogy could be defined with respect to our notion of faithful interpretation.

In contrast to the approach in this paper, Sowa's lattice of theories contains all possible first-order theories in all possible signatures. Most importantly, Sowa does not distinguish between conservative and non-conservative extension within this lattice of theories; consequently, no distinction can be made between the ordering over theories within a hierarchy and the ordering over theories between hierarchies.

Another approach that utilizes metatheoretic relationships among ontologies is HETS [20]. Using category theory as a mathematical basis, HETS defines the notions of conservative extension and definable interpretation between theories. In this sense, HETS could provide an alternative architecture to implement the ideas in this paper, although HETS does not have a relationship that is equivalent to reducibility. In addition, the notion of an ontology module within HETS [19] emphasizes the role of conservative extension.

\section{Two Characterizations of Modularity}

Repositories as we defined them lend themselves to two notions of modularity, one defined using similarities and another defined using reducibility. We conclude this section by showing how they complement each other in modularizing an ontology.

Notice that the resulting modules are not linked to a specific axiomatization. In fact, we do not distinguish between two equivalent theories with different axiomatizations. Likewise, we do not distinguish two modules with different axiomatizations as long as they are logically equivalent. 


\subsection{Similarity}

As we saw in Theorem 1, any ontology that is a non-root core theory in a core hierarchy has a unique profile consisting of a set of trunk theories. This set provides a natural modularization of the ontology: each trunk theory is a module that encapsulates an ontological design choice that the core theory shares with another theory in the same core hierarchy. All other theories in the hierarchy are combinations of the trunk theories, and additional modules arise to capture the similarities between different combinations.

Of course, the trunk theories are combined in a non-conservative way, which differs from typical approaches to modularity such as [9]. Nevertheless, the use of trunk theories for the notion of primitive module fits quite well with the intuition that they capture the smallest possible theory which can be shared and reused among multiple ontologies.

\subsection{Reducibility}

If an ontology is not a core theory in the repository, then the notion of trunk theory alone is insufficient to specify the modules within the ontology. In this case, we can use the notion of reducibility to decompose the ontology into modules that are definably equivalent to core theories within the repository.

Lemma 11 If a theory $T$ is reducible to a set of core theories in a repository $\mathbb{R}$, there exist core theories $S_{1}, \ldots, S_{n}$ such that $T$ is reducible to $S_{1}, \ldots, S_{n}$ and each $S_{i}$ belongs to a different core hierarchy.

Proof: Suppose that there exist multiple theories $S_{11}, S_{12}$ within the same core hierarchy $\mathbb{C}_{1}$ such that $T$ is reducible to $S_{1}, \ldots, S_{11}, S_{12}, \ldots, S_{n}$.

By Theorem 6, $T$ is definably equivalent to $S_{1} \cup \ldots \cup S_{11} \cup S_{12} \cup \ldots \cup S_{n}$. In particular, $T$ faithfully interprets both theories:

$$
\begin{gathered}
T \models \sigma \Leftrightarrow S_{11} \models \pi_{1}(\sigma) \\
T \models \sigma \Leftrightarrow S_{12} \models \pi_{1}(\sigma)
\end{gathered}
$$

Since we also have

$$
S_{1} \cup \ldots \cup S_{11} \cup S_{12} \cup \ldots \cup S_{n} \models \sigma \Leftrightarrow T \models \pi(\sigma)
$$

we know that $S_{11}$ and $S_{12}$ are logically equivalent.

If the repository contains multiple equivalent core hierarchies, then the reduction will contain multiple definably equivalent core theories, and hence there will exist multiple reductions that contain different sets of core theories.

Definition 22 A minimal reduction of a theory $T$ in a repository $\mathbb{R}$ is a set of theories $S_{1}, \ldots, S_{n}$ such that $S_{i}$ is not definably equivalent to $S_{j}$ for any $1 \leq i, j \leq n$ such that $i \neq j$. 
The key result of this section shows how we can use the reduction of a complex theory to decompose it into a set of subtheories that are irreducible modules.

Theorem 9 If $S_{1}, \ldots, S_{n}$ is a minimal reduction of a theory $T$ in a repository $\mathbb{R}$, then there exist subtheories $T_{1}, \ldots, T_{n}$ of $T$ such that

- $T \models T_{i}$;

- $T_{i}$ is definably equivalent to $S_{i}$.

Proof: If $S_{1}, \ldots, S_{n}$ is a minimal reduction of a theory $T$ in a repository $\mathbb{R}$, then $T$ is definably equivalent to $S_{1} \cup \ldots \cup S_{n}$ by Theorem 6 .

There exists an interpretation $\pi$ of $S_{1} \cup \ldots \cup S_{n}$ into $T$, so that for any $\sigma \in \mathcal{L}\left(S_{1} \cup\right.$ $\left.\ldots \cup S_{n}\right)$,

$$
S_{1} \cup \ldots \cup S_{n} \models \sigma \Leftrightarrow T \models \pi(\sigma)
$$

For each $S_{i}$, there exists an interpretation $\pi_{i}$ of $T$ into $S_{i}$, so that for any $\sigma \in \mathcal{L}(T)$

$$
T \models \sigma \Leftrightarrow S_{i} \models \pi_{i}(\sigma)
$$

Let

$$
T_{i}=\left\{\sigma: S_{i} \models \pi_{i}(\sigma), T \models \sigma\right\}
$$

By the definition of reducibility, $T$ faithfully interprets $S_{i}$, so that we have

$$
S_{i} \models \sigma \Leftrightarrow T \models \pi(\sigma)
$$

so that $T_{i}$ is definably equivalent to $S_{i}$.

We will refer to the set of subtheories $T_{1}, \ldots, T_{n}$ as the modularization of the theory $T$ in $\mathbb{R}$. The subtheory $\left(T \backslash \bigcup T_{i}\right)$ is referred to as the residue of $T$ in the modularization.

Note that even if there exist multiple reductions that contain different sets of core theories, each reduction leads to the same modularization, since the different theories in the reductions are definably equivalent. As we saw with the notion of similarity, we also need to draw attention to the fact that the modularization is a relationship between subtheories of $T$ and not simply between subsets of axioms of $T$.

\subsection{Synthesis}

The two notions of module presented in this section are compared in Table 1. Together they provide complementary approaches to both ontology modularity as well as ontology repositories. Any ontology can first be decomposed by finding the set of core theories that constitute its reduction. Each of these core theories can then be decomposed into the set of trunk theories in its profile. The first modularization step yields a set of theories that characterize the models of the ontology up to elementary equivalence, while the second modularization step supports a characterization of sharability and reuse with other ontologies. 


\begin{tabular}{|l|l|l|l|}
\hline Relationship between Theories & Set of Theories & Atomic Module & Modularization \\
\hline \hline non-conservative extension & hierarchy & trunk theory & profile \\
\hline reducibility & repository & core theory & reduction \\
\hline
\end{tabular}

Table 1. Comparison between the two notions of modularity discussed in this paper.

This approach also addresses the problem of the potentially exponential number of modules, discussed in [3]. If we consider trunk theories in a hierarchy and core theories within the reduction of a theory to be the building blocks of ontologies, then the number of such modules is bounded by the number of axioms within the theory. Of course, this can lead to an exponential set of theories in the repository if we explicitly construct all possible combinations of trunk theories and core theories. In the remainder of this paper, we investigate a series of procedures which decompose any ontology into a set of core theories, and which identify the set of trunk theories which are equivalent to a core theory. The only theories that we require to explicitly be included within the repository are those required by core hierarchies being closed and atomistic.

\section{Using the Repository}

The applications of our notion of ontology repository revolve around two perspectives on the intuitions underlying the notion of "module". In the first perspective, we decompose a first-order ontology into a set of disjoint subtheories which intuitively correspond to the modules of the ontology. The second perspective considers each trunk theory within a core hierarchy to be a module that axiomatizes a distinct ontological design choice within the setting of that core hierarchy. This notion of module is used to determine the relationships between theories with respect to the similarities and differences among them. The two perspectives on modularity motivate several scenarios that typify the application of an ontology repository in supporting the modularization of ontologies. The remainder of this paper will present semi-automated procedures that address these scenarios.

In the first scenario, we begin with a new ontology and determine whether or not it is reducible to a set of core theories within the repository. This captures the first perspective on modularity; as we will show in the Decomp Procedure (Section 6.4), reducibility can be used to identify those subtheories of the new ontology which are its modules. If a new ontology is not reducible, then there are two possibilities. In the optimistic case, the ontology is reducible to a set of theories which are extensions of core theories within the repository; once identified, the associated core hierarchies need to be updated with the new core theories. In the pessimistic case, the new ontology is a theory in a new core hierarchy, albeit one in which we have little understanding as to the intended models of its constituent theories. This case is essentially one of ontology design insofar as the verification of the ontology requires new representation theorems (since the characterization of the models cannot be reduced to the models of any existing ontologies in the repository).

The second scenario addresses an auxiliary theme of this paper, namely that ontology repositories are dynamic ontology design artefacts, and as such need to have the capability of being updated with new theories. The Update Procedure (Section 7.1) specifies exactly how one updates an existing core hierarchy with a new core theory while 
propagating the changes so that the resulting hierarchy is closed and atomistic: every non-root theory in the hierarchy is the union of a set of trunk theories of the hierarchy (compare Def. 11). The new core theory can either arise from the modularization procedure in the first scenario, or it can simply be a new theory that has been proposed within the research community.

Requiring all core hierarchies to be closed and atomistic allows us to easily determine the similarities and differences among various core theories within a single core hierarchy. As we have already seen, the similarities between core theories correspond to the ontological design choices that they share (i.e. the trunk theories in their shared profiles) and the differences correspond to the design choices for which the theories disagree. The third scenario generalizes this idea to complex hierarchies, that is, it uses the relationships between hierarchies to determine the similarities between complex theories within the same complex hierarchy.

These scenarios assume that we already have a repository with a set of (closed and atomistic) core hierarchies and maybe some complex hierarchies as well. However, one might ask how we actually can get to this stage. How can we initially populate the repository? In fact, this case can be reduced to the other two scenarios.

We first need to identify all hierarchies. By comparing the roots of any two hierarchies, we can decide which hierarchies will initially be core hierarchies. This is not covered by the procedures, but we can determine it by finding the similarity between any roots of two hierarchies.

We can start with one of the roots of each such hierarchy and successively add the other roots while ensuring that the hierarchy is closed and atomistic. Any non-root core theory can then be inserted using the Update Procedure. Once this process completes, all core hierarchies are in the repository and are closed and atomistic.

Finally, the non-core hierarchies are inserted one at a time using the Decomp Procedure, potentially creating additional core theories.

\section{Modularizing an Ontology}

In this section, we present a semi-automated procedure that uses the repository to decompose an ontology $T$ into a set of irreducible modules $T_{1}, \ldots, T_{n}$. The basis for this procedure is Theorem 9 - we first find a set of core theories which form the reduction of $T$, and then we specify the subtheories of $T$ which are definably equivalent to the core theories.

\subsection{Translation Definitions}

Recall that a set of theories $S_{1}, \ldots, S_{n}$ is a reduction of $T$ iff $T$ is definably equivalent to $S_{1} \cup \ldots \cup S_{n}$. The first step in the modularization of $T$ will be to recast the metatheoretic relationship of relative interpretation into an entailment problem. 
Definition 23 Let $T_{0}$ be a theory with signature $\Sigma\left(T_{0}\right)$ and let $T_{1}$ be a theory with signature $\Sigma\left(T_{1}\right)$ such that $\Sigma\left(T_{0}\right) \cap \Sigma\left(T_{1}\right)=\emptyset$.

Translation definitions for $T_{0}$ into $T_{1}$ are conservative definitions in $\Sigma\left(T_{0}\right) \cup \Sigma\left(T_{1}\right)$ of the form

$$
\forall \bar{x} p_{i}(\bar{x}) \equiv \Phi(\bar{x})
$$

where $p_{i}(\bar{x})$ is a relation symbol in $\Sigma\left(T_{0}\right)$ and $\Phi(\bar{x})$ is a formula in $\mathcal{L}\left(T_{1}\right)$.

Following [24], translation definitions can be considered to be an axiomatization of the interpretation of $T_{0}$ into $T_{1}$.

Theorem $10 T_{1}$ is interpretable in $T_{2}$ iff there exist a set of translation definitions $\Delta$ for $T_{1}$ into $T_{2}$ such that

$$
T_{2} \cup \Delta \models T_{1}
$$

Proof: If $T_{1}$ is interpretable in $T_{2}$, then there exists an interpretation $\pi$ such that $\pi$ assigns to each n-place relation symbol $P$ a formula $\pi_{P}$ of $\mathcal{L}\left(T_{1}\right)$ in which at most the variables $v_{1}, \ldots, v_{n}$ occur free [5].

It is easy to see that $\pi_{P}$ is the antecedent of a translation definition and that the literal $P\left(v_{1}, \ldots, v_{n}\right)$ is the consequent of a translation definition.

Conversely, suppose we are given a set of translation definitions $\Delta$ such that $T_{2} \cup \Delta=T_{1}$, that is,

$$
T_{1} \models \sigma \Rightarrow T_{2} \cup \Delta \models \sigma
$$

For each translation definition,

$$
\forall \bar{x} p_{i}(\bar{x}) \equiv \Phi_{i}(\bar{x})
$$

specify the mapping

$$
\pi\left(p_{i}\right)=\Phi_{i}(\bar{x})
$$

For any sentence $\sigma \in \mathcal{L}\left(T_{1}\right)$, we know that $\pi(\sigma)$ is logically equivalent to $\sigma$ by substitution of the consequents of the translation definitions in $\Delta$, so that

$$
T_{2} \cup \Delta \models \pi(\sigma)
$$

Since the translation definitions are conservative, this is equivalent to

$$
T_{2} \models \pi(\sigma)
$$

and hence $\pi$ is an interpretation. 
Theorem $11 T_{1}$ is faithfully interpretable in $T_{2}$ iff there exist a set of translation definitions $\Delta$ for $T_{1}$ into $T_{2}$ such that $T_{2} \cup \Delta$ is a conservative extension of $T_{1}$.

Proof: Theorem 10 shows that $T_{2} \cup \Delta$ is an extension of $T_{1}$.

Let $\pi$ be the interpretation associated with the translation definitions $\Delta$.

$T_{2} \cup \Delta$ is a conservative extension of $T_{1}$ iff for every sentence $\sigma \in \mathcal{L}\left(T_{1}\right)$,

$$
T_{2} \cup \Delta \models \sigma \Rightarrow T_{1} \models \sigma
$$

which holds iff

$$
T_{2} \models \pi(\sigma) \Rightarrow T_{1} \models \sigma
$$

which is equivalent to $\pi$ being a faithful interpretation

If we recall the definition of reducibility, we need to find a set of theories such that $T$ is definably equivalent to $T_{1} \cup \ldots \cup T_{n}$. The definition of definable equivalence requires the solution of two distinct sets of entailment problems:

$$
\begin{gathered}
T \cup \Delta_{i} \models T_{i} \\
T_{1} \cup \ldots \cup T_{n} \cup \Pi \models T
\end{gathered}
$$

where $\Delta_{i}$ is the set of translation definitions from $T$ to the theory $T_{i}$, and $\Pi$ is the set of translation definitions from the combined theory $T_{1} \cup \ldots \cup T_{n}$ to $T$.

The next lemma shows that the same translation definitions can be used for all theories that are stronger than some theory in the same hierarchy.

Lemma 12 Let $\pi$ be an interpretation of the theory $T_{1}$ in the hierarchy $\mathbb{H}_{1}$ into the theory $T_{2}$ in the hierarchy $\mathbb{H}_{2}$. Let $\Delta$ be the set of translation definitions for $\pi$.

If

$$
T_{1} \cup \Delta \models T_{2}
$$

and $T_{1}<T_{11}$, then $\mathbb{H}_{2}$ can be extended with a theory $T_{21}$ such that $T_{2}<T_{21}$ and

$$
T_{11} \cup \Delta \models T_{21}
$$

Proof: It is easy to see that

$$
\left(T_{21} \backslash T_{2}\right)=\left\{\pi(\sigma): T_{11} \models \sigma, \quad T_{2} \not \models \pi(\sigma), T_{21} \models \pi(\sigma)\right\}
$$

In general, translation definitions cannot be automatically generated for two arbitrary theories, since it is not decidable to determine if an interpretation between the two theories exists. 


\subsection{Translations of Axioms}

Rather than use the translation definitions as additional axioms used to entail the sentences in another theory, we can also specify a theory in which predicates in one signature are replaced by their definitions in the other signature.

Definition 24 Let $\Delta$ be the set of translation definitions $\Delta$ for the theory $T_{1}$ into the theory $T_{2}$.

The translation $T_{1}^{r}$ of $T_{1}$ is the set of sentences in $\mathcal{L}\left(T_{2}\right)$ in which each predicate in $T_{1}$ has been replaced by the consequent of its translation definition in $\Delta$.

As an example, we can consider one of the theories in the reduction of the theory $T_{p s l c o r e}$. The theory of linear orderings contains the sentence

$$
(\forall x, y, z) \operatorname{leq}(x, y) \wedge \operatorname{leq}(y, z) \supset \operatorname{leq}(x, z)
$$

Using the translation definition into the theory $T_{\text {pslcore }}$ :

$$
(\forall x, y) \operatorname{leq}(x, y) \equiv \text { before } E q(x, y)
$$

the translation of the sentence is

$$
(\forall x, y, z) \text { before } E q(x, y) \wedge \text { before } E q(y, z) \supset \text { before } E q(x, z)
$$

This technique of using the translations of sentences will play a key role in the decomposition procedure introduced later in this section.

It is a straightforward corollary of Theorem 10 that the translation $T_{1}^{r}$ of $T_{1}$ is logically equivalent to a subtheory of $T_{2}$.

Corollary 2 Let $\Delta$ be the set of translation definitions $\Delta$ for the theory $T_{1}$ into the theory $T_{2}$.

If $T_{1}^{r}$ is the translation of $T_{1}$, then

$$
T_{2} \models T_{1}^{r}
$$

\subsection{Finding Reducible Theories}

The FindTheory $(\mathbb{R}, T, \mathcal{T})$ Procedure addresses the first set of entailment problems by finding the set $\mathcal{T}$ of maximal core theories in the repository $\mathbb{R}$ that are interpretable by the theory $T$.

Beginning with the root theory in a core hierarchy $\mathbb{C}$, the procedure searches ${ }^{7}$ through $\mathbb{C}$ to find the maximal theories that are interpretable by $T$. If there are multiple maximal theories, the procedure returns the union of their sets of axioms.

\footnotetext{
${ }^{7}$ We assume the existence of three other algorithms related to partial orderings (see [2]). The first is ChainDecomp $(P)$ which outputs the set of chains for a poset $P$, and the second is $\operatorname{PosetSort}(X, P)$, which constructs a poset $P$ from an unordered set $X$. The third algorithm is $\operatorname{NextTheory}(T, P)$ which returns the elements in the poset $P$ that covers the element $T$.
} 
It is important to notice that there are two steps within this procedure that require user interaction. First, someone must determine whether or not the root theory of a particular core hierarchy is interpretable by $T$. Second, the entailment problem in step 11 typically requires human guidance even with an automated theorem prover.

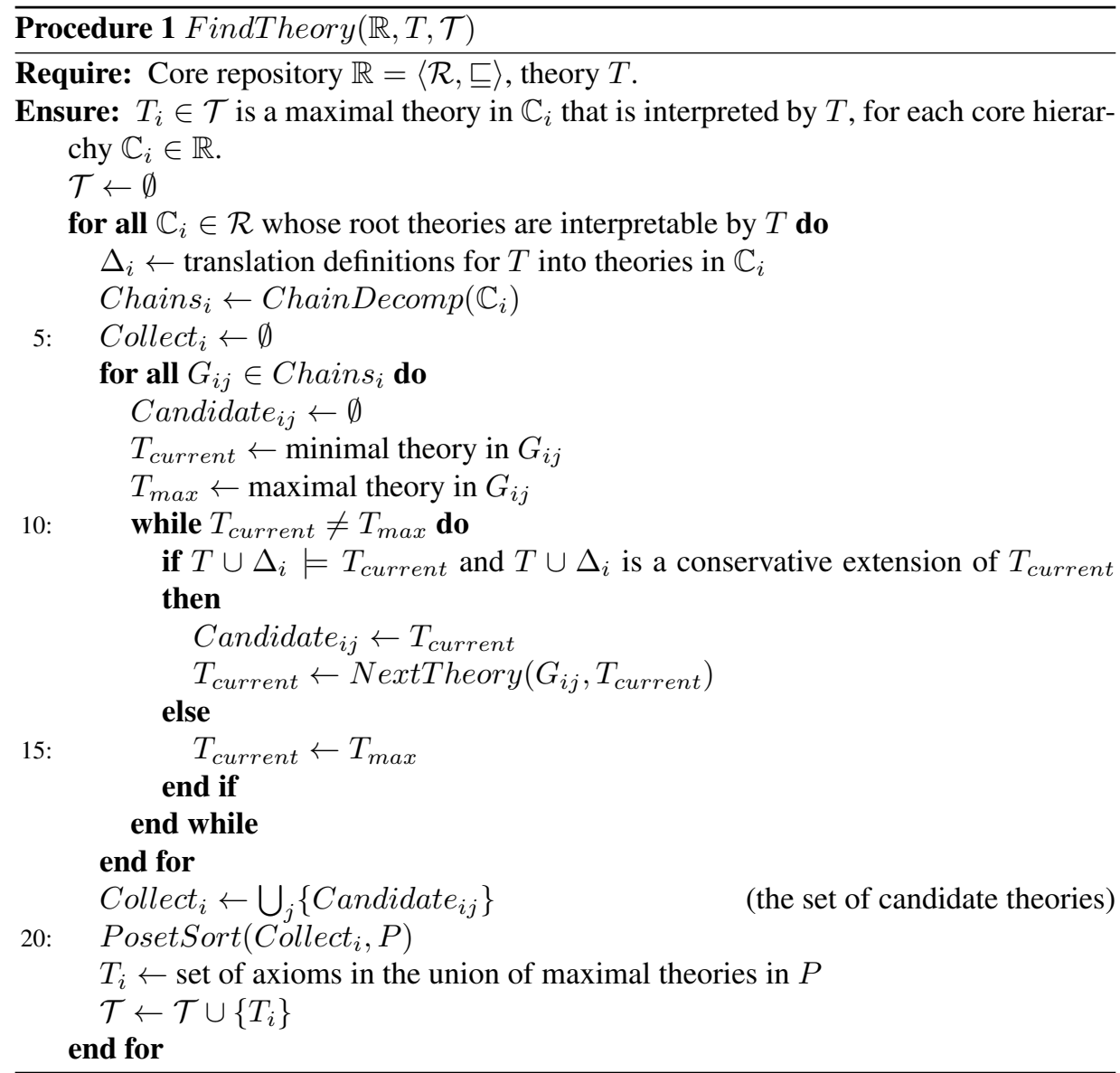

We turn now to the correctness of the FindTheory Procedure.

By the following lemma, if a theory $T_{i}$ in a core hierarchy $\mathbb{C}_{i}$ is not interpretable by $T$, then no extension of it in $\mathbb{C}_{i}$ is interpretable by $T$. In particular, if the root theory of $\mathbb{C}_{i}$ is not interpretable by $T$, then no theory in $\mathbb{C}_{i}$ is interpretable by $T$.

Lemma 13 Let $\mathbb{C}=\langle\mathcal{C}, \leq\rangle$ be a core hierarchy such that $T_{1}, T_{2} \in \mathcal{C}$. If $T_{2}$ is interpretable by $T$ and $T_{1}<T_{2}$, then $T_{1}$ is interpretable by $T$.

Proof: By Theorem 10 and Theorem 11, $T_{2}$ is interpretable by $T$ iff there exist translation definitions $\Delta$ such that

$$
T \cup \Delta \models T_{2}
$$

and $T \cup \Delta$ is a conservative extension of $T_{2}$. 
Since $T_{1}<T_{2}$, the definition of hierarchy gives us $T_{2} \models T_{1}$ and hence

$$
T \cup \Delta \models T_{1}
$$

so that $T_{1}$ is interpretable by $T$.

Lemma 14 Let $\mathbb{C}=\langle\mathcal{C}, \leq\rangle$ be a closed core hierarchy.

For any theory $T$, there exists at most one maximal theory in $\mathbb{C}$ that is interpretable by $T$.

Proof: Since $\mathbb{C}$ is closed, it has a unique root theory.

By Lemma 13, if the root theory of $\mathbb{C}$ is not interpretable by $T$, then no extension of the root theory is interpretable by $T$. Since all theories in $\mathbb{C}$ are extensions of the root theory, no theories in $\mathbb{C}$ are interpretable by $T$.

If there are multiple maximal theories $T_{1}, \ldots, T_{n}$ that are interpretable by $T$, then there exist translation definitions $\Delta$ such that

$$
T \cup \Delta \models T_{1} \cup \ldots \cup T_{n}
$$

so that $T_{1} \cup \ldots \cup T_{n}$ is a consistent extension of each $T_{i}$ which is interpretable by $T$ and is itself a theory in $\mathbb{C}$

Theorem 12 Let $\mathbb{R}=\langle\mathcal{R}, \sqsubseteq\rangle$ be a repository, and let $T$ be a theory such that $T \notin \mathcal{R}$.

If the FindTheory $(\mathbb{R}, T, \mathcal{T})$ Procedure terminates, then $T_{i} \in \mathcal{T}$ is a maximal theory in $\mathbb{C}_{i}$ that is interpretable by $T$, for each core hierarchy $\mathbb{C}_{i} \in \mathbb{R}$.

Proof: The procedure has the following loop invariant:

L Candidate $_{i j}$ is a theory in a chain in the core hierarchy $\mathbb{C}_{i}$ that is interpreted by $T$.

The root theory of $\mathbb{C}_{i}$ is the unique minimal theory in each chain in $\mathbb{C}_{i}$, and by the condition in Line 1, the root theory is interpretable by $T$. The loop invariant is therefore satisfied by the preconditions of the procedure.

We next show that the loop invariant is preserved at the end of the iteration. Candidate $_{i j}$ is updated in Line 12. By Lemma 12, the same translation definitions are used for all theories in the same hierarchy. By Theorem 10, Theorem 11, and the entailment problem in Line 11, if $T \cup \Delta_{i}=T_{\text {current }}$, then $T_{\text {current }}$ is faithful interpretable by $T$.

If $T \cup \Delta_{i} \not \models T_{\text {current }}$, then by Theorem 10, $T_{\text {current }}$ is not interpretable by $T$. By Lemma 13, no extension of $T_{\text {current }}$ is interpretable by $T$. In this case, the loop terminates and Candidate $_{i j}$ is the maximal theory in the chain that is interpretable by $T$.

By the assignment in Line 18, Collect $_{i}$ is the set of maximal theories in each chain in $\mathbb{C}_{i}$. The external Procedure PosetSort assigns to $P$ the maximal elements in Collect $_{i}$. By Lemma $14, T_{i}$ is the unique maximal theory in $\mathbb{C}_{i}$ that is interpretable by $T$.

Finally, we can see that if the entailment problem in Step 11 terminates, then the procedure terminates, since PosetSort terminates and each core hierarchy is finite (and hence has minimal and maximal elements), so that the loops will eventually terminate. 


\subsection{Ontology Decomposition Procedure}

In this section, we outline a semi-automated procedure for using the relationships among core theories and complex theories within the repository to decompose an ontology into irreducible modules.

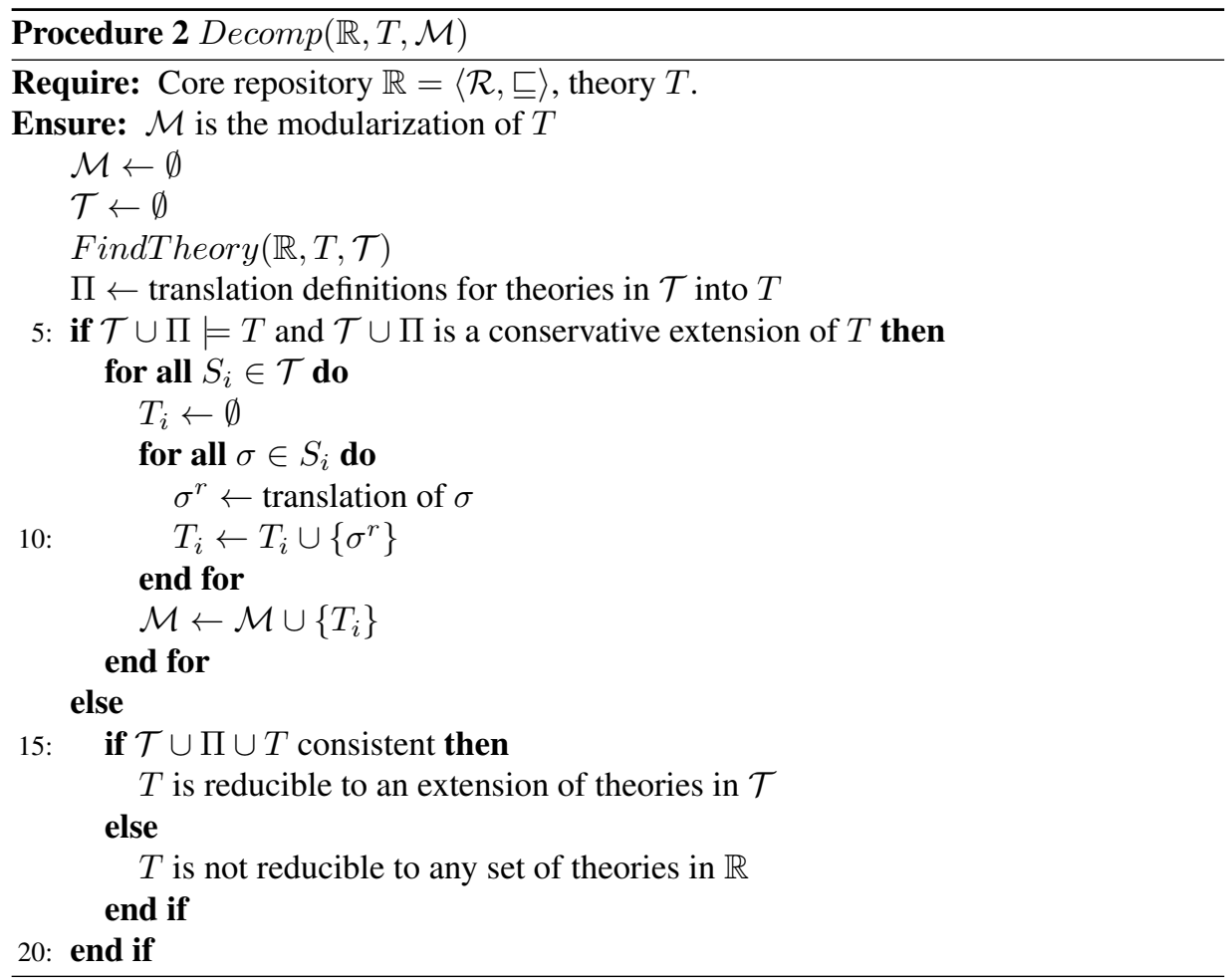

The FindTheory Procedure finds a set of core theories which are interpretable by the theory $T$. In order to show that a subset of these theories constitutes a reduction of $T$, we need to show that the union of such theories interprets $T$.

As discussed above, a user is required to provide the translation definitions $\Pi$ for the core theories $S_{1} \cup \ldots \cup S_{n}$ into $T$. If in fact we have $S_{1} \cup \ldots \cup S_{n} \cup \Pi \models T$, then by Theorem 8, we know that a modularization exists. Each module $T_{i}$ of $T$ is the subtheory of $T$ which is equivalent to the core theory $S_{i}$; we therefore determine which axioms within $T$ are entailed by $S_{i} \cup \Pi$ for each core theory within the reduction of $T$.

If an ontology is not reducible to the set of theories in a repository, we can use the notion of weak interpretability [4] to determine whether it is possible to extend the repository to include new core theories in existing core hierarchies such that a reduction exists in the new repository.

Definition 25 A theory $T_{1}$ is weakly interpretable in theory $T_{2}$ iff there is a function on $\Sigma\left(T_{1}\right)$ such that for any sentence $\sigma \in \mathcal{L}\left(T_{1}\right)$,

$$
T_{1} \models \sigma \Rightarrow T_{2} \not \models \neg \pi(\sigma)
$$


The results of [4] show that a theory $T_{1}$ is weakly interpretable in a theory $T_{2}$ iff $T_{1}$ is interpretable in some consistent extension of $T_{2}$.

Lemma $15 T$ is interpretable in some consistent extension of $S_{1} \cup \ldots \cup S_{n}$ iff there exist translation definitions $\Pi$ such that

$$
S_{1} \cup \ldots \cup S_{n} \cup \Pi \cup T
$$

is consistent.

Proof: $T$ is interpretable in some consistent extension of $S_{1} \cup \ldots \cup S_{n}$ iff there exist translation definitions $\Pi$ such that

$$
S_{1}^{\prime} \cup \ldots \cup S_{n}^{\prime} \cup \Pi \models T
$$

where $S_{i} \subseteq S_{i}^{\prime}$, from which it follows that $S_{1} \cup \ldots \cup S_{n} \cup \Pi \cup T$ is consistent.

This Lemma is the justification for lines 15-19 in the Decomp Procedure.

In general, the extension of the repository is an ontology design task. Under restricted conditions, however, we can identify new core theories. For example, suppose we have three core theories $S_{11}, S_{12}$, and $S_{21}$ such that $S_{11}<S_{12}$ and

$$
\begin{gathered}
T \cup \Delta_{1} \cup \Delta_{2} \models S_{21} \cup S_{11} \\
S_{21} \cup S_{11} \cup \Pi \not \models T
\end{gathered}
$$

but

$$
\begin{gathered}
S_{21} \cup S_{12} \cup \Pi \models T \\
T \cup \Delta_{1} \cup \Delta_{2} \not \models S_{21} \cup S_{12}
\end{gathered}
$$

By Lemma 15, we know that there exists a theory $S_{13}$ such that $S_{11}<S_{13}$ and

$$
\begin{gathered}
T \cup \Delta_{1} \cup \Delta_{2} \models S_{21} \cup S_{13} \\
S_{21} \cup S_{13} \cup \Pi \models T
\end{gathered}
$$

and hence that $T$ is reducible to $S_{21} \cup S_{13}$.

An additional problem is that if $S_{1} \cup \ldots \cup S_{n} \cup \Pi \not \models T$, multiple core theories may need to be extended, but we do not a priori know which ones need to be extended.

If a theory $T$ is not reducible to any theories within the repository, then effectively $T$ itself is the first theory in a new hierarchy of its own. We say that such a theory is under probation; as the etymology suggests, additional proofs are needed to determine the relationship between this new theory and existing theories within the repository. In particular, new representation theorems are required to establish the verification of the new theory. 
Theorem 13 Let $\mathbb{R}=\langle\mathcal{R}, \sqsubseteq\rangle$ be a repository, and let $T$ be a theory such that $T \notin \mathcal{R}$.

If $T$ is reducible to a set of theories in the repository and the Decomp $(\mathbb{R}, T, \mathcal{M})$ Procedure terminate, then $\mathcal{M}$ is a modularization of $T$.

Proof: By Theorem 12, FindTheory $(\mathbb{R}, T, \mathcal{T})$ returns a set of theories $\mathcal{T}$ in $\mathbb{R}$ that are interpretable by $T$.

There are three cases:

1. $\mathcal{T} \cup \Pi=T$;

2. $\mathcal{T} \cup \Pi \not \models T$ but $\mathcal{T} \cup \Pi \cup T$ consistent;

3. $\mathcal{T} \cup \Pi \cup T$ is not consistent.

Case 1: (Line 5) By Theorem 6, $T$ is reducible to the theories in $\mathcal{T}$, that is, $\mathcal{T}$ is a reduction of $T$.

By Theorem 9, there exist subtheories $T_{1}, \ldots, T_{n}$ of $T$ such that each $T_{i}$ is definably equivalent to some $S_{i} \in \mathcal{T}$.

The outer loop (Lines 6-13) iterates through the theories in $\mathcal{T}$. The inner loop (Lines 8-11) iterates through the axioms in $S_{i} \in \mathcal{T}$. $T_{i}^{r}$ is the set of translations of axioms in $S_{i}$. By Corollary $2, T_{i}^{r} \subseteq T$ and and $T_{i}^{r}$ is definably equivalent to $S_{i}$.

The assignment in Line 12 collects the subtheories of $T$.

Case 2: (Line 15) By Lemma 15, $T$ is reducible to extensions of the theories in $\mathcal{T}$. Case 3: (Line 18) By Lemma 15, $T$ is not reducible to any core theories in $\mathbb{R}$.

It is important to stress that Decomp and FindTheory are procedures and not algorithms, both because of the undecidable theorem proving and consistency-checking steps (Line 9 in FindTheory and Lines 5 and 15 in Decomp), but also because of the user intervention required for the specification of translation definitions (Line 2 of FindTheory and Line 4 of Decomp). In this sense, the procedures give practical guidance for the designers of modular ontologies.

\section{Updating a Hierarchy}

In the preceding section, we observed that if an ontology is not reducible to a set of theories already contained in a repository, then the repository can be extended to include new theories.

Here we present a semi-automated procedure that inserts a new theory into a closed, atomistic repository so that the resulting repository is still closed and atomistic. This procedure is intended to be used with the core hierarchies which are required to be closed and atomistic in our repository, but is not restricted to those. In general, this leads to the introduction of new trunk theories into the (core) hierarchy, as well as the refinement of some former trunk theories in the original (core) hierarchy.

Adding a theory $T$ to an existing closed, atomistic hierarchy presumes, of course, that the signature of $T$ is the same as the signature of the theories in the hierarchy, and that $T$ is an extension of the root theory of the hierarchy. Procedures 3, 4, and 5 give the full details. The following notation is used throughout the procedures:

$\operatorname{root}(\mathbb{C}):$ Reference to the root theory of the closed, atomistic hierarchy $\mathbb{C}$.

$\operatorname{trunk}(\mathbb{C})$ : Reference to the set of trunk theories of the closed, atomistic hierarchy $\mathbb{C}$. 
profile $(T)$ : Reference to the set of theories that constitute the profile of the theory $T$. $\operatorname{similarity}\left(T_{1}, T_{2}\right)$ : Returns a theory $T$ that is the similarity of the theories $T_{1}$ and $T_{2}$ in the sense of Definition 7.

difference $\left(T_{1}, T_{2}\right)$ : Returns a theory $T$ that is the difference between the theories $T_{1}$ and $T_{2}$ in the sense of Definitions 13 and 14.

When adding a theory $T$ to a closed, atomistic hierarchy, there are three possibilities:

1. $T$ is logically equivalent to an existing core theory;

2. $T$ is logically equivalent to the union of a set of trunk theories in the hierarchy (i.e. the profile of $T$ );

3. There does not exist a subset of the trunk theories whose union entails $T$.

Procedure 3 UpdateHierarchy $(\mathbb{C}, T)$

Require: A closed, atomistic hierarchy $\mathbb{C}=\langle\mathcal{C}, \leq\rangle$, and a theory $T$ such that $\Sigma(\mathcal{C})=$ $\Sigma(T)$ and $T \models \operatorname{root}(\mathbb{C})$.

Ensure: $\mathbb{C}=\langle\mathcal{C} \cup\{T\}, \leq\rangle$ is a closed, atomistic hierarchy.

UpdateProfile $(\mathbb{C}, T)$

$\mathcal{C} \leftarrow \mathcal{C} \cup\{T\}$

CloseHierarchy $(\mathbb{C}, T)$

Procedure 4 UpdateProfile $(\mathbb{C}, T)$

Require: A closed, atomistic hierarchy $\mathbb{C}=\langle\mathcal{C}, \leq\rangle$, a theory $T$ such that $\Sigma(\mathcal{C})=\Sigma(T)$ and $T=\operatorname{root}(\mathbb{C})$.

Ensure: $T$ has a profile in $\mathbb{C}, \mathbb{C}$ is atomistic and the similarity between any two trunk theories is a root theory.

Covered $\leftarrow \emptyset$

$T^{d} \leftarrow T \quad$ (the difference between $T$ and the currently covered trunk theories)

while $\exists T^{\prime} \in \operatorname{trunk}(\mathbb{C}) \backslash$ Covered with similarity $\left(T^{\prime}, T^{d}\right) \neq \operatorname{root}(\mathbb{C})$ do

$S \leftarrow \operatorname{similarity}\left(T^{\prime}, T^{d}\right)$

5: $\quad$ Covered $\leftarrow$ Covered $\cup\{S\}$

$T^{d} \leftarrow$ difference $\left(T^{d}, S\right)$

$T^{\prime d} \leftarrow$ difference $\left(T^{\prime}, S\right)$

$\operatorname{trunk}(\mathbb{C}) \leftarrow\left(\operatorname{trunk}(\mathbb{C}) \backslash T^{\prime}\right) \cup\left\{S, T^{\prime d}\right\}$

profile $\left(T^{\prime}\right) \leftarrow\left\{S, T^{\prime d}\right\}$

10: $\quad$ for all $T_{i} \in \mathcal{C}$ do

if $T^{\prime} \in \operatorname{profile}\left(T_{i}\right)$ then

profile $\left(T_{i}\right) \leftarrow\left(\operatorname{profile}\left(T_{i}\right) \backslash T^{\prime}\right) \cup \operatorname{profile}\left(T^{\prime}\right)$

end if

end for

15: end while

$\operatorname{trunk}(\mathbb{C}) \leftarrow \operatorname{trunk}(\mathbb{C}) \cup\left\{T^{d}\right\}$

profile $(T) \leftarrow$ Covered $\cup\left\{T^{d}\right\}$ 


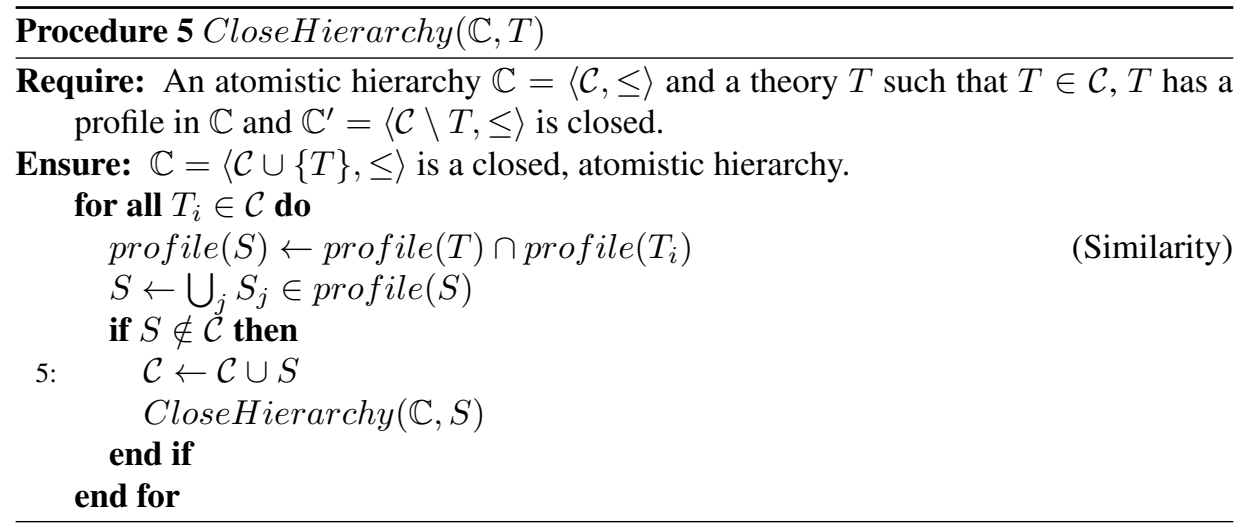

In the first two cases, the profile of the new theory $T$ consists entirely of already existing trunk theories. In the first case, the execution of UpdateHierarchy results in no change in the hierarchy. In the second case, the execution of the procedure amounts to identification of the profile of the new theory and the similarities and differences of the theory with other non-trunk theories.

The third case is more interesting, since it means that a new trunk theory must be added to the core hierarchy $\mathbb{C}$ in order to maintain an atomistic hierarchy. This is handled by the UpdateProfile Procedure which inserts all necessary new trunk theories into the hierarchy and determines which (if any) existing trunk theories are no longer trunk theories. As a side effect, UpdateProfile determines the new profile for every existing theory in the hierarchy. In particular, the difference $T^{d}$ of $T$ with respect the union of all trunk theories entailed by $T$, i.e. $\bigcup\left\{T^{\prime} \mid T^{\prime} \in \operatorname{trunk}(\mathbb{C}) \wedge T \models T^{\prime}\right\}$, is itself a theory in the same hierarchy by the definition of differences. Moreover, $T_{\text {comp }}$ is independent of the other trunk theories that are in the profile of $T$. If there is no nontrivial similarity between $T^{d}$ and the trunk theories (i.e. the similarity is the root theory), then $T^{d}$ covers (see Def. 9) the root theory and is itself a new trunk theory. On the other hand, if $T^{d}$ does have a nontrivial similarity $S$ with one of the existing trunk theories $T^{\prime}$, then $T^{\prime}$ no longer covers the root theory and hence it is no longer a trunk theory. In addition, the difference between $T^{\prime}$ and $S$ is also a subtheory of $T^{\prime}$ and the difference between $T^{d}$ and $S$ is also a subtheory of $T^{d}$. Now $S$ and both differences are trunk theories in the hierarchy - unless we can find further similarities with other trunk theories.

For the first two cases, that is, if no new trunk theory needs to be added, we only need to update the similarities to ensure closure. By Theorem 1 this can be achieved using the profiles alone (see CloseHierarchy) because the hierarchy is still atomistic. The UpdateHierarchy Procedure terminates in all three cases if all calls of similarity and difference terminate. In the following, we prove this property as well as correctness, i.e. that the resulting hierarchy $\mathbb{C}=\langle\mathcal{C} \cup T, \leq\rangle$ is closed (under similarities) and atomistic. 
Lemma 16 Let $\mathbb{C}=\langle\mathcal{C}, \leq\rangle$ be a closed, atomistic hierarchy and let $T$ be a theory such that $\Sigma(\mathcal{C})=\Sigma(T)$ and $T \models \operatorname{root}(\mathbb{C})$. If the UpdateProfile $(\mathbb{C}, T)$ Procedure terminates, the resulting hierarchy $\mathbb{C}_{\text {new }}=\left\langle\mathcal{C}_{\text {new }}, \leq\right\rangle$ is atomistic and $T$ has a profile in $\mathbb{C}_{\text {new }}$.

Proof: We begin by stating the loop invariants of the procedure:

$\mathbf{L 1} \bigcup_{i}$ Covered $_{i} \cup\left\{T^{d}\right\}=T$

L2 The similarity between any two trunk theories is the root theory.

$\mathbf{L 3} \cup_{i}$ Covered $_{i}$ has a profile in $\mathbb{C}$.

L4 $\mathbb{C}$ is atomistic.

Let the preconditions of the procedure be $\rho$ and let $T^{d}=T$ and Covered $=\emptyset$ as set before entering the loop. Then,

$\rho \Rightarrow L 1$ : Since $\bigcup_{i}$ Covered $_{i}=\emptyset$ and $T^{d}=T$

$\rho \Rightarrow L 2$ : By Lemma 4

$\rho \Rightarrow L 3$ : The profile of $\bigcup_{i}$ Covered $_{i}$ is the empty profile.

$\rho \Rightarrow L 4$ : Trivial.

Next we show that given the loop invariants hold before an iteration, they are preserved at the end of the iteration.

$L 1$ : Line 5 adds a theory $S$ to the Covered and line 6 updates $T^{d}$ to be the difference between the previous $T^{d}$ and $S . L 1$ follows from the definitions of remainder and difference.

$L 2$ : Trunk theories are updated in line 8. The condition holds since $T^{\prime}=S \cup\left\{T^{\prime d}\right\}$ and the similarity between $S$ and $T^{\prime d}$ is the root theory as $T^{\prime d}$ is the difference between $T^{\prime}$ and $S$ (line 7).

L3: The only theory that is added to Covered is $S$. Since $S$ is added to the trunk theories in line 8 , Covered has a profile in $\mathbb{C}$.

L4: The only way this condition can be broken is if a non-trunk theory is created that does not have a profile in $\mathbb{C}$. Line 8 , is the only operation in the procedure that creates a non-trunk theory by removing $T^{\prime}$ from $\operatorname{trunk}(\mathbb{C})$. Since $T^{\prime}=S \cup$ $\left\{T^{\prime d}\right\}$ and both $S$ and $T^{\prime d}$ are added to $\operatorname{trunk}(\mathbb{C})$ in the same line, the condition is preserved.

Once the loop condition is false, $L 1, L 2, L 3, L 4$ and the following hold:

$\forall T^{\prime} \in \operatorname{trunk}(\mathbb{C})\left[\operatorname{similarity}\left(T^{\prime}, T^{d}\right)=\operatorname{root}(\mathbb{C})\right]$

After the execution of lines 16 and 17 we obtain the postconditions:

T has a profile in $\mathbb{C}$ by $L 1, L 3, \lambda$ and line 17 .

$\mathbb{C}$ is atomistic by $L 4$.

The similarity condition by $\mathrm{L} 2, \lambda, 16$.

Finally, we show that if the similarity and difference functions terminate, the procedure terminates. At each iteration the selected trunk theory is split into two parts: the similarity $S$ which is added to Covered and $T^{\prime d}$ whose similarity with $T^{d}$ is the root theory. Therefore neither of the new trunk theories will be selected again. Since the hierarchy contain only finitely many theories and thus have only a finite number of trunk theories, the loop will eventually terminate. 
Lemma 17 Let $\mathbb{C}=\langle\mathcal{C}, \leq\rangle$ be an atomistic hierarchy and let $T$ be a theory such that $T \in \mathcal{C}, T$ has a profile in $\mathbb{C}$ and $\mathbb{C}^{\prime}=\langle\mathcal{C} \backslash T, \leq\rangle$ is closed. After executing the CloseHierarchy $(\mathbb{C}, T)$ Procedure the resulting hierarchy $\mathbb{C}_{\text {new }}=\left\langle\mathcal{C}_{\text {new }}, \leq\right\rangle$ is closed and atomistic.

Proof: The procedure amounts to finding all theories that have similarities with $T$, adding the similarities to the hierarchy if they do not already exist and repeating the procedure for all the newly added similarities. It is straightforward to see that if the procedure terminates the hierarchy will be closed. We observe that a discovered similarity is the intersection of the profiles of two existing theories in the hierarchy. Since each theory in the hierarchy is distinct, the newly added theories have profiles that include fewer trunk theories than their parents. Therefore, since the hierarchy has a finite number of trunk theories, at some point no new similarities can be discovered and the procedure terminates.

Note that, in general there may be a theory $T^{\prime}$ in $\mathcal{C}$ such that $T$ is inconsistent with $T^{\prime}$. Since $T$ is an extension of the root theory of the hierarchy and the hierarchy is closed, a non-empty similarity between $T$ and $T^{\prime}$ is guaranteed to exist. In other words, $T$ and $T^{\prime}$ will at least share the root theory as their similarity, hence the closed property of the hierarchy will be preserved.

Theorem 14 Let $\mathbb{C}=\langle\mathcal{C}, \leq\rangle$ be a closed, atomistic hierarchy and let $T$ be a theory such that $\Sigma(\mathcal{C})=\Sigma(T)$ and $T \models \operatorname{root}(\mathbb{C})$. If the UpdateHierarchy $(\mathbb{C}, T)$ Procedure terminates, the resulting hierarchy $\mathbb{C}_{\text {new }}=\left\langle\mathcal{C}_{\text {new }}, \leq\right\rangle$ is closed, atomistic and $T$ has a profile in $\mathbb{C}_{\text {new }}$.

Proof: Follows directly from Lemmas 16 and 17

Consider the core hierarchy in Figure 5 as example. It consists of two trunk theories $T_{\text {dense_nofinal_partial }}$ and $T_{\text {discrete_noinitial_partial }}$ that share only the root theory $T_{\text {partial_ordering }}$ as their similarity. Now we would like to add a new core theory, $T_{\text {inf_ordering }}$, to this hierarchy while maintaining that the core hierarchy is closed and atomistic. In this scenario, the theory we are adding, $T_{\text {inf_ordering }}$, is not equivalent to any combination of core theories in the hierarchy. Therefore, UpdateProfile is run in order to determine the new subtheory that must be added to the hierarchy to ensure that $T_{\text {inf_ordering }}$ is the union of a set of trunk theories. Since $T_{\text {inf_ordering }}$ shares similarities with each trunk theory, the procedure breaks down those trunk theories into two subtheories each (one being the similarity shared with $T_{\text {inf_ordering }}$ and the other being the difference between the old trunk theory and the similarity). Here, the similarity between $T_{\text {inf_ordering }}$ and $T_{\text {dense_nofinal_partial }}$ is $T_{\text {nofinal }}$, and the difference between $T_{\text {dense_nofinal_partial }}$ and the new similarity is $T_{\text {dense_partial }}$. For the other trunk theory, we get the new subtheories $T_{\text {noinitial }}$ and $T_{\text {discrete_partial }}$. It then follows from the definition of trunk theories that these new subtheories are added to the set of trunk theories while the original trunk theories that cover them are no longer considered as trunk theories. Each time a subtheory is added to the hierarchy, it is also added to the profile of the original theory. Here, subtheories $T_{\text {noinitial }}$ and $T_{\text {discrete_partial }}$ were added to the profile of $T_{\text {discrete_nointial_partial }}$ at the same time they were added as trunk theories in the hierarchy. Finally, the subtheory of $T_{\text {inf_ordering }}$ that shares no non-trivial 


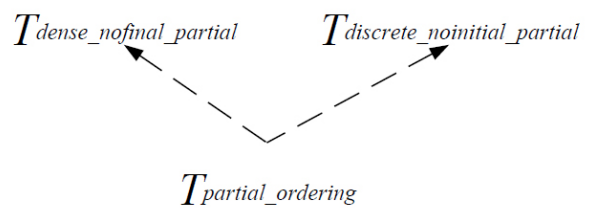

Figure 5. Initial hierarchy with two trunk theories extending the root theory. Dashed lines denote non-conservative extensions.

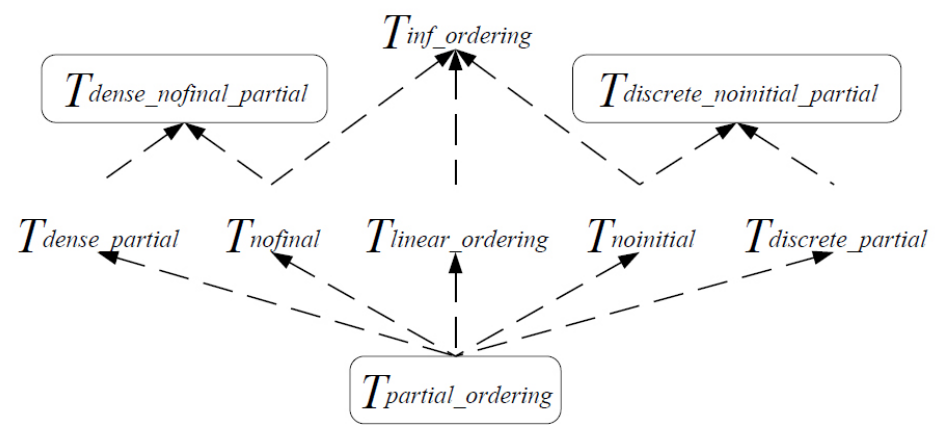

Figure 6. Hierarchy after a new theory $T_{i n f_{-} \text {ordering }}$ is added using UpdateHierarchy. Dashed lines denote non-conservative extensions. Theories that existed in the hierarchy before insertion of the new theory are outlined.

similarity with any trunk theory ( $T_{\text {linear_ordering }}$ ) is added to the set of trunk theories. UpdateProfile completes and the next step CloseHierarchy makes no changes for this example as the hierarchy at this step happens to be closed under similarity. Figure 6 shows the hierarchy after UpdateHierachy is completed, with the original theories before the procedure was run outlined.

\section{Towards an Implementation in COLORE}

All of the techniques and metatheoretic relationships between theories discussed in this paper are being developed in the context of the COLORE (Common Logic Ontology Repository) project, which is building an open repository of first-order ontologies that serve as a testbed for ontology evaluation and integration techniques, and that can support the design, evaluation, and application of ontologies in first-order logic. All ontologies are specified using Common Logic (ISO 24707), which is a recently standardized logical language for the specification of first-order ontologies and knowledge bases.

At the lowest level are theories of general mathematical structures, such as algebraic structures (e.g. semigroups, groups, rings, vector spaces), and combinatorial structures (e.g. orderings, lattices, graphs). These ontologies serve as the basis for the reducibility of generic ontologies currently within the repository, such as processes, time, mereotopology, and geometry.

The relation $<$ between theories within the same hierarchy and the relation of conservative extensions of theories in different hierarchies are both equivalent to the clo- 
sure of $\mathrm{cl}$-module within COLORE under the cl-imports relation. The notion of reducibility and the $\sqsubseteq$ relation between hierarchies will require extensions to Common Logic.

Recent work in the verification of time ontologies has demonstrated the utility of the procedures introduced in this paper. In [21], the relationships between three different hierarchies of time interval ontologies are explored. New ontologies arose from definable equivalence between hierarchies and similarities within each hierarchy. In [14] and [10], a hierarchy was constructed that combined several ontologies that axiomatize both timepoints and time intervals. One interesting discovery was that the similarity of two ontologies proposed in [16] was actually equivalent to the time ontology independently axiomatized in [25].

\section{Summary}

This paper has explored the deep connection between ontology modularity and the logical relationships between theories within an ontology repository. Our formal characterization of repository revolves around the notion of hierarchies. Hierarchies are sets of theories that have the same non-logical lexicon; theories within the same hierarchy are related by non-conservative extension. The hierarchies themselves are related by reducibility, by which a theory in a complex hierarchy is definably equivalent to a set of theories in core hierarchies. For each of these relationships, we can define a class of theories that correspond to the intuition of modules. Within hierarchies, the atomic modules are known as trunk theories, which can be considered as a formalization of the ontological design choices for the hierarchy. On the level of repositories, the ordering over hierarchies defines core theories, which are building blocks for more complex ontologies.

Based on these complementary approaches to modularity, we present two sets of semi-automated procedures that support the decomposition of an ontology into modules. The first set of procedures identifies modules as subtheories of the ontology that are definably equivalent to some core theories and that are related to the ontology through reducibility. The second set of procedures takes any core theory and constructs the set of trunk theories whose combination is logically equivalent to the core theory. In addition, this set of trunk theories allows a characterization of which subtheories are shareable and reusable between ontologies.

This work naturally leads to the following challenges:

- Develop techniques for finding new core theories in the case when a reduction of a new theory $T$ does not exist, but $T$ is reducible to an extension of the core hierarchies.

- Incorporate techniques for ontology verification to characterize the models of new theories that are under probation as a result of the Decomp Procedure.

- Explore techniques that use the reductions and profiles of theories to generate semantic mappings between ontologies.

- In any hierarchy, the complete set of trunk theories for a core hierarchy corresponds to the axioms of all complete extensions of the root theory. Can these trunk theories in turn be used to design new extensions of existing ontologies that are reducible to the core hierarchies? 


\section{Acknowledgement}

We are grateful to the three reviewers for their detailed comments which helped to improve the clarity of the presentation of the paper.

\section{References}

[1] Burstall, R.M. and Goguen, J.A. (1977) Putting theories together to make specifications. International Joint Conference on Artificial Intelligence 1977, pp. 1045-1058.

[2] Daskalakis, C., Karp, R., Mossel, E., and Riesenfeld, S. (2009) Sorting and Selection in Posets. Proc. of the Twentieth Annual ACM-SIAM Symposium on Discrete Algorithms, pp. 392-401.

[3] Del Vescovo, C., Parsia, B., Sattler, U., and Schneider, T. (2009) The Modular Structure of an Ontology: An Empirical Study. Proc. of the Fourth Int. Workshop on Modular Ontologies, IOS Press.

[4] Dzhaparidze, G. (1993) A Generalized Notion of Weak Interpretability and the Corresponding Modal Logic. In: Annals of Pure and Applied Logic 61:113-160.

[5] Enderton, H. (1972) Mathematical Introduction to Logic, Academic Press.

[6] Farmer, W. M. (2000) An Infrastructure for Intertheory Reasoning. Proc. of the Seventeenth Int. Conference on Automated Deduction (CADE-17), LNCS 1831, pp. 115-131.

[7] Farmer, W. M., Guttman, J. D., Thayer, F. J. (1992) Little Theories. Proc. of the Eleventh Int. Conference on Automated Deduction (CADE-11), LNCS 607, pp. 567-581.

[8] Cuenca Grau, B., Parsia, B., Sirin, E., Kalyanpur, A. Automatic Partitioning of OWL Ontologies Using E-Connections. Proc. of the Int. Workshop on Description Logics (DL2005), 2005.

[9] Cuenca Grau, B., Parsia, B., Sirin, E. (2009) Ontology Integration Using E-Connections. Modular Ontologies: Concepts, Theories and Techniques for Knowledge Modularization, LNCS 5445, pp. 159-186.

[10] Grüninger, M. (2011) Verification of the OWL-Time Ontology, in Proceedings of the Tenth International Semantic Web Conference. Bonn, Germany.

[11] Grüninger, M. (2009) Definability and Process Ontologies. Proc. of the Ninth Int. Symposium on Logical Formalizations of Commonsense Reasoning, Toronto, Canada.

[12] Grüninger, M. (2009) Process Ontologies. In: Handbook of Ontologies, 2nd Edition, S. Staab (ed.), pp. 419-421, Springer.

[13] Grüninger, M., Hahmann, T., Hashemi, A., and Ong, D. (2010) Ontology Verification with Repositories. Proc. of the Sixth Int. Conference on Formal Ontologies in Information Systems (FOIS 2010), pp. 317330, IOS Press.

[14] Grüninger, M. and Ong, D. (2011) Verification of Time Ontologies with Points and Intervals, in 18th International Symposium on Temporal Representation and Reasoning, Lübeck, Germany.

[15] Hashemi, A. (2009) Using Repositories for Ontology Design and Semantic Mapping, MASc thesis, University of Toronto, Toronto, Canada.

[16] Hayes, P. (1996) A Catalog of Temporal Theories, Tech Report UIUC-BI-AI-96-01, Univ. Illinois.

[17] Hodges, W. (1993) Model Theory. Cambridge University Press.

[18] Konev, B., Lutz, C., Walther, D., and Wolter, F. (2009) Formal Properties of Modularisation. Modular Ontologies: Concepts, Theories and Techniques for Knowledge Modularization, LNCS 5445, pp. 25-66.

[19] Kutz, O. and Mossakowski, T. (2008) Conservativity in structured ontologies. In Proceedings of the European Conference on Artificial Intelligence 2008. IOS Press, Amsterdam.

[20] Mossakowski, T., Maeder, C., Lüttich, K., Wölfl, S. (2004) The Heterogeneous Tool Set. Available at www.tzi.de/cofi/hets, University of Bremen.

[21] Ong, D. and Grüninger, M. (2011) Constructing an Ontology Repository: A Case Study with Theories of Time Intervals, in Proceedings of the Fifth International Workshop on Modular Ontologies. Lljubljana.

[22] Schorlemmer, M. and Kalfoglou, Y. (2008) Institutionalising Ontology-Based Semantic Integration, Applied Ontology 3:131-150.

[23] Sowa, J. (2000) Knowledge Representation: Logical, Philosophical, and Computational Foundations, Brooks/Cole Publishing Co., Pacific Grove, CA.

[24] Szczerba, L.W. (1977) Interpretability of Elementary Theories. In: Logic, Foundations of Mathematics and Computability Theory, Butts and Hintikka (eds.), pp. 129-145, Springer.

[25] Vila, L. (2005) Formal Theories of Time and Temporal Incidence, in Handbook of Temporal Reasoning in Artificial Intelligence, Fisher, Gabbay, Vila: eds. Elsevier, 2005. 